\title{
MOLECULAR STRUCTURE, VIBRATIONAL SPECTRA, NBO, FUKUI FUNCTION, HOMO-LUMO ANALYSIS AND MOLECULAR DOCKING STUDY OF 6-[(2-METHYLPHENYL)SULFANYL]-5-PROPYLPYRIMIDINE-2,4(1H,3H)-DIONE
}

\author{
Haitham Al-Rabiah ${ }^{1}$, S. Muthu ${ }^{2}$, Fatmah A. M. Al-Omary ${ }^{1}$, Abdul-Malek S. Al-Tamimi ${ }^{3}$, \\ M. Raja ${ }^{4}$, R. Raj Muhamed ${ }^{4}$, Ali A. El-Emam ${ }^{1 *}$ \\ ${ }^{1}$ Department of Pharmaceutical Chemistry, College of Pharmacy, King Saud University, \\ Riyadh 11451, Saudi Arabia \\ ${ }^{2}$ Department of Physics, Arignar Anna Govt. Arts College, Cheyyar, 604407, Tamilnadu, India \\ ${ }^{3}$ Department of Pharmaceutical Chemistry, College of Pharmacy, Prince Sattam bin Abdulaziz University, \\ Alkharj 11942, Saudi Arabia \\ ${ }^{4}$ Department of Physics, Jamal Mohamed College, Tiruchirappalli 620020, Tamilnadu, India \\ *aelemam@ksu.edu.sa; elemam5@hotmail.com
}

Theoretical and experimental FT-IR and FT-Raman vibrational spectral analysis of 6-[(2methylphenyl) sulfanyl]-5-propylpyrimidine-2,4(1H,3H)-dione have been recorded in the region 4000$400 \mathrm{~cm}^{-1}$ and $4000-100 \mathrm{~cm}^{-1}$ in solid phase. The molecular geometric parameters bond length, bond angle and vibrational wave numbers, as well as harmonic vibrational frequency were investigated using the density functional theory B3LYP method with the $6-311++\mathrm{G}(\mathrm{d}, \mathrm{p})$ basis set. The stability of the molecule has been investigated using the natural bond orbital (NBO) analysis. The electronic properties such as HOMO-LUMO energies were determined by the time-dependent DFT approach. The thermodynamic properties and the first order hyperpolarizability and molecular electrostatic potential (MEP) of the title compound were also studied. The electron density-based local reactivity descriptors such as the Fukui functions were calculated to explain the chemical selectivity or reactivity site in the molecule. The molecule orbital contributions were investigated using the total density of states (TDOS), the sum of $\alpha$ and $\beta$ electron density of states ( $\alpha \beta$ DOS). The molecular docking (ligand-protein) simulations have been performed using the AutoDock 4.2.6. Binding energy, bonded residues and donor-acceptor bond length values revealed that title compound can act as potential inhibitor against HIV-1 protease.

Keywords: FT-IR; FT-Raman; NBO; MEP; Fukui function; pyrimidine-2,4(1H,3H)-dione; molecular docking

\section{МОЛЕКУЛСКА СТРУКТУРА, ВИБРАЦИОНИ СПЕКТРИ, NВО, ФУНКЦИЈА НА ФУКУИ, НОМО-LUМО АНАЛИЗА И ИСПИТУВАҢЕ НА МОЛЕКУЛСКО ПРИПОЈУВАЫЕ НА 6-[(2-МЕТИЛФЕНИЛ)СУЛФАНИЛ]-5-ПРОПИЛПИРИМИДИН-2,4-(1Н,3Н)-ДИОН}

Теориските и експерименталните анализи на FT-IR и FT-Раманови вибрациони спектри на 6-[(2-метилфенил)сулфанил]-5-пропилпиримидин-2,4-(1H,3H)-дион беа снимени во областа од $4000-400 \mathrm{~cm}^{-1}$ и $4000-100 \mathrm{~cm}^{-1}$ во цврста фаза. Молекулските геометриски параметри на должини на врски, агли на врски и вибрациони бранови броеви, како и хармониските вибрациони фреквенции беа испитувани со помош на методот B3LYP на теоријата на функционалот на електронската густина со базисниот сет 6-311++G(d,p). Стабилноста на молекулата беше испитана со помош на анализа на природните сврзувачки орбитали (NBO). Електронските својства како што се енергиите на HOMO-LUMO беа определени со временски зависниот DFT пристап. Исто така беа истражувани термодинамичките својства и хиперполаризабилноста од прв ред и молекулскиот електростатски потенцијал (МЕР) на ова соединение. Дескрипторите на локалната реактивност 
засновани на електронската густина како што се функциите на Фукуи беа пресметани со цел да се објасни хемиската селективност или реактивните места во молекулата. Придонесите на молекулските орбитали беа изучувани со употреба на состојбите на вкупната густина (TDOS), како збир на $\alpha$ и $\beta$ состојбите на електронската густина $(\alpha \beta \mathrm{DOS})$. Симулациите на молекулско припојување (лиганд-протеин) беа извршени со помош на AutoDock 4.2.6. Сврзувачката енергија, сврзаните остатоци, како и вредностите на донорно-акцепторните должини на врски укажуваат дека ова соединение може да биде потенцијален инхибитор на протеазата на HIV-1.

Клучни зборови: FT-IR; FT на Раман; NBO; MEP; функција на Фукуи; пиримидин-2,4(1H,3H)-дион; молекулско припојување

\section{INTRODUCTION}

Pyrimidine and its related derivatives like uracil and thymine occupy a distinct position in the field of chemotherapy. Several pyrimidine and pyrimidine-related drugs are currently employed for the treatment of various diseases. Numerous pyrimidine-based derivatives have been developed as antiviral agents against human immunodeficiency viruses (HIV) [1-5], hepatitis B viruses (HBV) [6, 7], hepatitis $\mathrm{C}$ viruses (HCV) [8] and herpes simplex viruses (HSV) $[9,10]$. In addition, several pyrimidine derivatives have long been utilized as potent anticancer drugs [11-14]. Moreover, potent antibacterial [15-19], fungicidal [20, 21] and antiprotozoal activities [22-25] were recognized by several pyrimidine derivatives. 1-[(2-Hydroxyethoxy)methyl]-6-(phenylthio)thymine (HEPT) and its related analogues [26-29] were discovered as potent and selectively active agents against HIV-1 infections. In continuation of our ongoing interest in the pharmacological and structural properties of pyrimidine and uracil derivatives $[3,4,17,18,30$ 35], we synthesized the title compound as the more lipophilic 5-propyl HEPT analog for evaluation as a potential chemotherapeutic agent [36].

The FT-IR-Raman spectroscopy combined with quantum chemical computations have been recently used as an effective tool in the vibrational analysis of drugs. The search for new materials with nonlinear optical (NLO) properties has been the subject of intense research due to their application in a wide range of technologies such as optical computing and communications [37, 38]; much attention has been paid to organic NLO materials due to their promising applications in optoelectronic technology [39, 40]. Vibrational spectral studies of molecules can provide more in depth knowledge about the relationships between molecular architecture, nonlinear response, and hyperpolarizability, supporting the efforts towards the discovery of new efficient materials for technological applications.
In the present study, the FT-IR and FTRaman spectral investigation of the title compound has been performed using the density functional theory (DFT). A complete vibrational analysis of the molecule was performed by combining the experimental and theoretical information using $\mathrm{Pu}-$ lay's DFT based scaled quantum mechanical (SQM) approach. The change in electron density (ED) in the $\sigma^{*}$ and $\pi^{*}$ anti-bonding orbitals and the stabilization energies $E(2)$ have been calculated by NBO analysis to acquire clear evidence of stabilization originating in the hyperconjugation of the hydrogen-bonded interaction. In addition, the electron density-based local reactivity descriptor such as the Fukui functions are calculated employing the Mulliken charges to explain the chemical selectivity or reactivity sites in the title molecule. The geometric structure, highest occupied molecular orbital (HOMO) energy, lowest unoccupied molecular orbital (LUMO) energy, and molecular electrostatic potential (MEP) analyses of the title compound were also performed to predict information regarding charge transfer within the molecule.

\section{EXPERIMENTAL DETAILS}

The title compound $(\mathbf{C})$ was prepared starting with 5-propylbarbituric (A) acid with phosphorus oxychloride and trace amounts of water to yield 5-propyl-6-chlorouracil (B), which was further reacted with $o$-thiocresol in ethanolic potassium hydroxide to yield the target compound (Scheme $1)$. The reaction was carried out via heating a mixture of 6-chloro-5-propyluracil (943 mg, 0.005 $\mathrm{mol}), o$-thiocresol $(621 \mathrm{mg}, 0.005 \mathrm{~mol})$ and potassium hydroxide $(281 \mathrm{mg}, 0.005 \mathrm{~mol})$, in ethanol $(10 \mathrm{ml})$, under reflux for three hours. The solvent was then distilled off in vacuo and the residue was washed with cold water, dried and recrystallized from ethanol to yield $940 \mathrm{mg}(68 \%)$ of the title compound $\left(\mathrm{C}_{14} \mathrm{H}_{16} \mathrm{~N}_{2} \mathrm{O}_{2} \mathrm{~S}\right)$ as colorless needle crystals. M.P.: $210-212{ }^{\circ} \mathrm{C}$. The structure of the title compound was established by ${ }^{1} \mathrm{H}$ NMR, ${ }^{13} \mathrm{C}$ NMR 
and single crystal X-ray diffraction [36]. ${ }^{1} \mathrm{H}$ NMR (DMSO-d 6 , $500.13 \mathrm{MHz}): \delta 0.84\left(\mathrm{t}, 3 \mathrm{H}, \mathrm{CH}_{2} \mathrm{CH}_{3}\right.$, $J=7.0 \mathrm{~Hz}), 1.37-1.40\left(\mathrm{~m}, 2 \mathrm{H}, \mathrm{CH}_{2} \mathrm{CH}_{3}\right), 2.33(\mathrm{~s}$, $\left.3 \mathrm{H}, \mathrm{Ar}-\mathrm{CH}_{3}\right), 2.43\left(\mathrm{t}, 2 \mathrm{H}, \mathrm{CH}_{2} \mathrm{CH}_{2} \mathrm{CH}_{3}, J=7.0\right.$ $\mathrm{Hz}), 6.92-7.02(\mathrm{~m}, 3 \mathrm{H}, \mathrm{Ar}-\mathrm{H}), 7.26-7.28(\mathrm{~m}, 1 \mathrm{H}$, Ar-H), $10.91(\mathrm{~s}, 1 \mathrm{H}, \mathrm{NH}), 11.24(\mathrm{~s}, 1 \mathrm{H}, \mathrm{NH}) .{ }^{13} \mathrm{C}$ NMR (DMSO-d $6, \quad 125.76 \quad \mathrm{MHz}): \quad \delta \quad 13.72$ $\left(\mathrm{CH}_{2} \mathrm{CH}_{3}\right), 22.06 \quad\left(\mathrm{CH}_{2} \mathrm{CH}_{3}\right), 20.12\left(\mathrm{Ar}-\mathrm{CH}_{3}\right)$, $28.22\left(\mathrm{CH}_{2} \mathrm{CH}_{2} \mathrm{CH}_{3}\right), 117.44$ (Pyrimidine $\mathrm{C}-5$ ), 125.90, 126.50, 129.88, 130.20, 133.18, 140.56 (Ar-C), 143.02 (Pyrimidine C-6), $150.53(\mathrm{C}=\mathrm{O})$, $163.23(\mathrm{C}=\mathrm{O})$.<smiles>CCCC1C(=O)NC(=O)NC1=O</smiles>

A

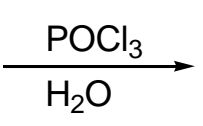<smiles></smiles><smiles>Cc1ccccc1S</smiles><smiles>CCCc1c(Sc2ccccc2C)[nH]c(=O)[nH]c1=O</smiles>

Scheme 1. Synthesis of the title compound

The FT-IR spectrum has been recorded in the region $4000-400 \mathrm{~cm}^{-1}$ in evacuation mode using $\mathrm{KBr}$ pellet technique with $1.0 \mathrm{~cm}^{-1}$ resolution on a Perkin Elmer FT-IR spectrophotometer. The FT-Raman spectrum was recorded in the region $4000-100 \mathrm{~cm}^{-1}$ in pure mode using Nd:YAG Laser of $100 \mathrm{~mW}$ with $2 \mathrm{~cm}^{-1}$ resolution on a Brucker RFS 27 Raman spectrophotometer.

\section{COMPUTATIONAL DETAILS}

The entire calculations were performed at the DFT (B3LYP) method 6-311++G(d,p) basis set on a personal computer using the Gaussian $03 \mathrm{~W}$ $[41,42]$ program package invoking gradient geometry optimization [43]. The optimized molecular structural parameters were used in the harmonic vibrational frequency. The IR Intensity and Raman activity were calculated using the B3LYP/6$311++\mathrm{G}(\mathrm{d}, \mathrm{p})$ basis set by combining the results of the Gauss-view program with the harmonic vibrational frequency assignments with a high degree of accuracy. The PEDs are computed from the quantum chemically-calculated vibrational frequencies using the VEDA program [44]. The natural bonding orbital (NBO) calculations were performed using the NBO 3.1 program [45] implemented in the Gaussian 03 package at the B3LYP /6$311++\mathrm{G}(\mathrm{d}, \mathrm{p})$ level in order to understand various second-order molecular interactions between the filled orbitals of one subsystem and the vacant orbitals of another subsystem, which is a measure of the intermolecular delocalization or hyperconjugation. Molecular docking (ligand-protein) simulations were performed using AutoDock 4.2.6. It should be noted that the Gaussian 03 package does not calculate the Raman intensity. The calculated Raman activities $\left(\mathrm{S}_{\mathrm{i}}\right)$ with the Gaussian 03W program have been converted to the relative Raman Intensities $\left(\mathrm{L}_{\mathrm{i}}\right)$ using the following relationship derived from the basis theory of Raman scattering [46, 47]:

$$
I_{i}=10^{-12}\left(\mathrm{n}_{0}-\mathrm{n}_{\mathrm{i}}\right)^{4}\left(1 / \mathrm{n}_{\mathrm{i}}\right) . S
$$

where $I_{i}$ is the Raman intensity, $S$ is the Raman scattering activities, $n_{i}$ is the wave number of normal modes, and $n_{o}$ denotes the wave number of the excitation laser.

\section{RESULTS AND DISCUSSION}

\subsection{Molecular geometry}

The optimized molecular geometry of the title compound was obtained from the Gaussian $03 \mathrm{~W}$ program with the atom numbering scheme shown in Figure 1. The comparative optimized structural parameters such as bond length, bond angle along with its experimental data [36] are presented in Table 1 . The most optimized geometry is performed at the B3LYP /6-311++G(d, P) basis set of the title molecule. The molecule has 35 atoms and 99 normal modes of fundamental vibrations. All 99 vibrations are active in both IR and Raman.

The theoretical values for the title molecule were found to be closely related to the experimental XRD values. The maximum bond lengths were calculated for the $\mathrm{C}_{5}-\mathrm{C}_{12}, \mathrm{~S}_{12}-\mathrm{C}_{13}$ and found to be $1.792 \AA, 1.792 \AA$ (experimental) and $1.787 \AA$, $1.801 \AA$ (theoretical), respectively. The calculated bond lengths $\mathrm{C}_{3}-\mathrm{C}_{4}(1.386 \AA, 1.462 \AA), \mathrm{C}_{4}-\mathrm{C}_{9}$ $(1.499 \AA, 1.507 \AA)$ are found to be slightly higher than the experimental values. There are some devi- 
ations when compared with the experimental data, and these differences are probably due to the intermolecular or intramolecular interactions in the solid state. The computed bond lengths and bond angles are in reasonable agreement with the corresponding experimental values. The experimental values of the bond angles correlate well to the theoretical values (DFT) in the solid phase. The minor deviation can be attributed to the fact that the theoretical calculations were aimed at the isolated molecules in the gaseous phase and the experimental results were aimed at the molecule in the solid state. Despite these differences, the calculated geometric parameters represent a good approximation, and are the basis for the calculation of other parameters such as polarizability, vibrational frequencies and thermodynamic properties.

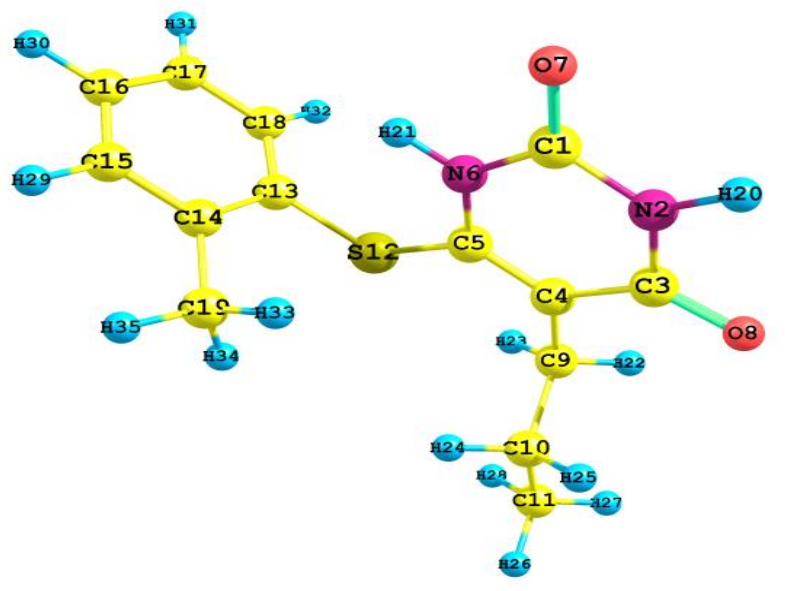

Fig. 1. Optimized ground state structure of the title compound (B3LYP/6-311++G(d,p)

Table 1

The optimized parameters (bond lengths and bond angle of the title compound

\begin{tabular}{ccc}
\hline \hline Parameters & B3LYP/6-311++G(d,p) & Experimental* \\
\hline 1 & $\mathbf{2}$ & $\mathbf{3}$ \\
\hline C1-N2 & Bond length $(\mathbf{A})$ & \\
C1-N6 & 1.378 & 1.362 \\
C1-O7 & 1.389 & 1.381 \\
N2-C3 & 1.214 & 1.223 \\
N2-H20 & 1.408 & 1.380 \\
C3-C4 & 1.012 & 0.830 \\
C3-O8 & 1.462 & 1.386 \\
C4-C5 & 1.220 & 1.223 \\
C4-C9 & 1.361 & 1.398 \\
C5-N6 & 1.507 & 1.499 \\
C5-S12 & 1.380 & 1.381 \\
N6-H21 & 1.787 & 1.792 \\
C9-C10 & 1.011 & 0.830 \\
C9-H22 & 1.541 & 1.534 \\
C9-H23 & 1.094 & 0.970 \\
C10-C11 & 1.095 & 0.097 \\
C10-H24 & 1.531 & 1.459 \\
C10-H25 & 1.096 & 0.970 \\
C11-H26 & 1.094 & 0.970 \\
C11-H27 & 1.093 & 0.970 \\
C11-H28 & 1.094 & 0.970 \\
S12-C13 & 1.095 & 0.970 \\
C13-C14 & 1.801 & 1.792 \\
C13-C18 & 1.411 & 1.521 \\
C14-C15 & 1.400 & 1.386 \\
C14-C19 & 1.386 \\
C15-C16 & 1.399 & 1.521 \\
C15-H29 & 1.386 \\
C16-C17 & 1.507 & 0.970 \\
C16-H30 & 1.386 \\
C17-C18 & 0.970 \\
C17-H31 & 1.393 & 1.386 \\
C18-H32 & 0.970 \\
C19-H33 & 1.085 & 0.970 \\
C19-H34 & 0.970 \\
C19-H35 & 1.392 & 0.970 \\
& 1.084 &
\end{tabular}




\begin{tabular}{|c|c|c|}
\hline 1 & 2 & 3 \\
\hline \multicolumn{3}{|c|}{ Bond angle $\left({ }^{\circ}\right)$} \\
\hline N2-C1-N6 & 112.8 & 114.51 \\
\hline $\mathrm{N} 2-\mathrm{C} 1-\mathrm{O} 7$ & 124.5 & 122.14 \\
\hline $\mathrm{C} 1-\mathrm{N} 2-\mathrm{C} 3$ & 127.5 & 126.26 \\
\hline $\mathrm{C} 1-\mathrm{N} 2-\mathrm{H} 20$ & 116.2 & 115.4 \\
\hline N6-C1-O7 & 122.7 & 123.35 \\
\hline C1-N6-C5 & 124.4 & 123.55 \\
\hline C1-N6-H21 & 115.4 & 115.4 \\
\hline C3-N2-H20 & 116.3 & 115.4 \\
\hline $\mathrm{N} 2-\mathrm{C} 3-\mathrm{C} 4$ & 115.4 & 116.35 \\
\hline $\mathrm{N} 2-\mathrm{C} 3-\mathrm{O} 8$ & 119.5 & 120.1 \\
\hline C4-C3-O8 & 125.1 & 123.4 \\
\hline C3-C4-C5 & 118.1 & 121.60 \\
\hline C3-C4-C9 & 117.4 & 117.4 \\
\hline C5-C4-C9 & 124.5 & 124.1 \\
\hline C4-C5-N6 & 121.8 & 121.6 \\
\hline C4-C5-S12 & 120.8 & 122.6 \\
\hline C4-C9-C10 & 113.6 & 117.4 \\
\hline C4-C9-H22 & 107.8 & 108.9 \\
\hline C4-C9-H23 & 110.3 & 109.3 \\
\hline N6-C5-S12 & 117.4 & 115.5 \\
\hline C5-N6-H21 & 120.2 & 120.4 \\
\hline C5-S12-C13 & 103.4 & 100.7 \\
\hline C10-C9-H22 & 108.8 & 108.9 \\
\hline C10-C9-H23 & 109.4 & 109.3 \\
\hline C9-C10-C11 & 112.4 & 117.4 \\
\hline C9-C10-H24 & 109.1 & 109.3 \\
\hline C9-C10-H25 & 108.7 & 108.9 \\
\hline $\mathrm{H} 22-\mathrm{C} 9-\mathrm{H} 23$ & 106.7 & 107.7 \\
\hline C11-C10-H24 & 109.8 & 109.3 \\
\hline C11-C10-H25 & 109.8 & 109.3 \\
\hline C10-C11-H26 & 111.2 & 109.3 \\
\hline C10-C11-H27 & 111.1 & 109.3 \\
\hline C10-C11-H28 & 111.3 & 109.3 \\
\hline H24-C10-H25 & 106.9 & 107.7 \\
\hline H26-C11-H27 & 107.7 & 107.7 \\
\hline H26-C11-H28 & 107.7 & 107.7 \\
\hline $\mathrm{H} 27-\mathrm{C} 11 \mathrm{H} 28$ & 107.7 & 107.7 \\
\hline S12-C13-C14 & 121.4 & 120.2 \\
\hline S12-C13-C18 & 117.5 & 118.7 \\
\hline C14-C13-C18 & 120.9 & 120.5 \\
\hline C13-C14-C15 & 117.3 & 117.3 \\
\hline C13-C14-C19 & 122.5 & 122.0 \\
\hline C13-C18-C17 & 120.4 & 120.6 \\
\hline C13-C18-H32 & 119.2 & 119.2 \\
\hline C15-C14-C19 & 120.2 & 120.0 \\
\hline C14-C15-C16 & 121.8 & 122.0 \\
\hline C14-C15-H29 & 118.7 & 119.7 \\
\hline C14-C19-H33 & 111.6 & 109.5 \\
\hline C14-C19-H34 & 111.2 & 109.5 \\
\hline C14-C19-H35 & 110.6 & 109.5 \\
\hline C16-C15-H29 & 119.5 & 119.7 \\
\hline C15-C16-H17 & 120.1 & 119.7 \\
\hline C15-H16-H30 & 119.7 & 119.7 \\
\hline C17-C16-H30 & 120.2 & 119.7 \\
\hline C16-C17-H18 & 119.4 & 119.7 \\
\hline C16-C17-H31 & 120.7 & 119.7 \\
\hline C18-C17-H31 & 120 & 119.7 \\
\hline C17-C18-H32 & 120.4 & 119.7 \\
\hline H33-C19-H34 & 106.9 & 109.5 \\
\hline H33-C19-H35 & 107.8 & 109.3 \\
\hline H34-C19-H35 & 108.6 & 109.3 \\
\hline
\end{tabular}

*Reference [36] 


\subsection{Vibrational assignments}

The title molecule consists of 35 atoms, which has 99 normal modes of vibration. The experimental FT-IR and FT-Raman spectra with their corresponding theoretically-simulated IR and Raman spectra are shown in Figures 2 and 3, respectively. The calculated vibrational frequencies (unscaled and scaled), IR intensity, and Raman activity are shown in Table 2.

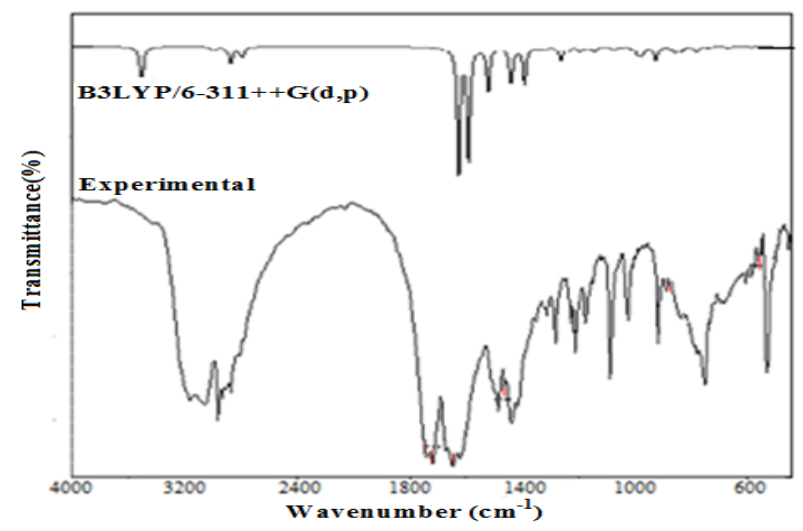

Fig. 2. Experimental and theoretical (B3LYP/6-311++G(d,p) FT- IR spectra of the title compound

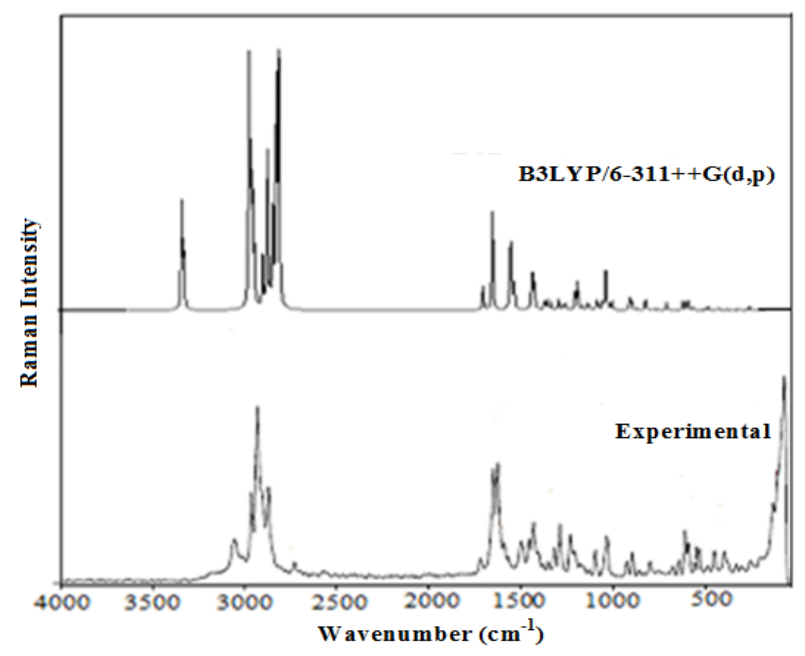

Fig. 3. Experimental and theoretical (B3LYP/6-311++G(d,p) FT- Raman spectra of the title compound

\subsection{1. $N$ - $H$ vibrations}

The N-H stretching vibrations of the heterocyclic compounds always occur in the region of $3500-3000 \mathrm{~cm}^{-1}$ [48-50]. The bands observed at 3440 and $3159 \mathrm{~cm}^{-1}$ in the FT-IR and at 3475, 3445 and $3076 \mathrm{~cm}^{-1}$ in the FT Raman spectra with $100,100,100,100$ and $97 \%$ of PED contribution are attributed to the $\mathrm{N}-\mathrm{H}$ groups of the pyrimidine ring. The theoretically-calculated values of the $\mathrm{N}$ $\mathrm{H}$ vibrations by the B3LYP/6-311++G(d,P) in high frequency region are reliable with the experimental results and these assignments are in good agreement with the literature data [35].

\subsection{2. $C$-C vibrations}

The ring $\mathrm{C}-\mathrm{C}$ aromatic vibrations give rise to characteristic bands in both the observed FT-IR and FT-Raman spectra covering the spectral range from 1600 to $1400 \mathrm{~cm}^{-1}$ [50]. In the present study, the C-C stretching vibration bands are calculated at 1591, 1522, 1497 and $1395 \mathrm{~cm}^{-1}$ in the FT-Raman spectra with major PED contributions of $11 \%$. The calculated bands at the B3LYP/6-311++G(d,P) levels are in excellent agreement with the experimental observations of both the FT-IR and FTRaman spectra of title compound. The ring C-C vibrations have given rise to weak bands across the low frequency region below $1320 \mathrm{~cm}^{-1}$. The bands at 1263,1178, 1155, 1090, 1047, 1010 and 535 $\mathrm{cm}^{-1}$ in the FT-IR and 1180, 1094, 1033 and 1003 $\mathrm{cm}^{-1}$ in the FT-Raman spectra have been assigned to the C-C stretching vibrations with $74,56,10$, $20,18,31,17$ and 56, 32, 17 and $92 \%$ contributions of PED, respectively.

\subsubsection{Aromatic $C-H$ vibrations}

The aromatic structure shows the presence of C-H stretching vibrations in the region 3100$3000 \mathrm{~cm}^{-1}$, which is the characteristic region for identification of the aromatic $\mathrm{C}-\mathrm{H}$ stretching vibrations [51]. In this region, the bands are not affected appreciably by the nature of the constituents. In the present investigation, the aromatic $\mathrm{C}-\mathrm{H}$ stretching vibrations were observed at 3073, 3063, 3054 and $3043 \mathrm{~cm}^{-1}$ by the B3LYP/6-311++G(d,P) method which showed excellent agreements with the bands observed in the recorded FT-Raman spectrum at $3076,3067,3054 \mathrm{~cm}^{-1}$ and with the FT-IR bands at $3159,3050 \mathrm{~cm}^{-1}$. The PED corresponding to this pure mode of contributing to $94 \%$, as shown in Table 2.

\subsubsection{Propyl vibrations}

The asymmetric and symmetric stretching modes of the propyl group in this compound are known to be influenced by a variety of interesting interactions such as electronic effects, intermolecular hydrogen bonding in the crystalline network and Fermi resonance [52]. 
T a ble 2

Observed and calculated vibrational frequency of the title compound at the B3LYP method with 6-311++G(d,P) basis set

\begin{tabular}{|c|c|c|c|c|c|c|c|}
\hline \multirow[t]{2}{*}{ Mode } & \multicolumn{2}{|c|}{$\begin{array}{c}\text { Experimental wave } \\
\text { number }\left(\mathrm{cm}^{-1}\right)\end{array}$} & \multicolumn{2}{|c|}{$\begin{array}{c}\text { Theoretical wave } \\
\text { number }\left(\mathrm{cm}^{-1}\right)\end{array}$} & \multirow{2}{*}{$\underset{\text { intensity }^{c}}{\text { IR }}$} & \multirow{2}{*}{$\underset{\text { intensity }^{b}}{\text { Raman }^{\prime}}$} & \multirow{2}{*}{$\begin{array}{l}\text { Vibrational assignments } \\
(\text { PED })^{\mathrm{a}}\end{array}$} \\
\hline & FT-IR & FT- Raman & Unscaled & Scaled $^{\mathrm{d}}$ & & & \\
\hline 1 & 2 & 3 & 4 & 5 & 6 & 7 & 8 \\
\hline 1 & - & 3475 & 3603 & 3462 & 9 & 43 & $\gamma \mathrm{NH}(100)$ \\
\hline 2 & 3440 & 3445 & 3592 & 3452 & 21 & 23 & $\gamma \mathrm{NH}(100)$ \\
\hline 3 & 3159 & 3076 & 3198 & 3073 & 1 & 100 & $\gamma \mathrm{CH}(97)$ \\
\hline 4 & - & 3067 & 3187 & 3063 & 1 & 40 & $\gamma \mathrm{CH}(87)$ \\
\hline 5 & - & 3054 & 3178 & 3054 & 1 & 42 & $\gamma \mathrm{CH}(100)$ \\
\hline 6 & 3050 & - & 3166 & 3043 & 0 & 19 & $\gamma \mathrm{CH}(93)$ \\
\hline 7 & - & - & 3116 & 2994 & 2 & 21 & $\gamma \mathrm{CH} 3(97)$ \\
\hline 8 & 3440 & 3445 & 3592 & 3452 & 21 & 23 & $\gamma \mathrm{NH}(100)$ \\
\hline 9 & - & 2980 & 3088 & 2968 & 7 & 17 & $\gamma \mathrm{CH} 3(84)$ \\
\hline 10 & - & - & 3086 & 2966 & 5 & 36 & $\gamma \mathrm{CH} 3(80)$ \\
\hline 11 & - & 2962 & 3086 & 2965 & 1 & 15 & $\gamma \mathrm{CH} 3(92)$ \\
\hline 12 & 2961 & - & 3075 & 2955 & 0 & 3 & $\gamma \mathrm{CH} 3(99)$ \\
\hline 13 & - & 2931 & 3054 & 2935 & 1 & 38 & $\gamma \mathrm{CH} 3(72)$ \\
\hline 14 & 2929 & - & 3034 & 2916 & 4 & 17 & $\gamma \mathrm{CH} 2(95)$ \\
\hline 15 & 2912 & - & 3033 & 2915 & 1 & 72 & $\gamma \mathrm{CH} 3(96)$ \\
\hline 16 & - & 2871 & 3021 & 2903 & 2 & 42 & $\gamma \mathrm{CH} 3(87)$ \\
\hline 17 & 1719 & 1719 & 1790 & 1721 & 100 & 10 & $\gamma \mathrm{OC}(74)$ \\
\hline 18 & 1662 & 1649 & 1734 & 1666 & 89 & 41 & $\gamma \mathrm{OC}(81)$ \\
\hline 19 & - & 1591 & 1629 & 1565 & 5 & 20 & $\gamma \mathrm{CC}(57)$ \\
\hline 20 & - & 1552 & 1622 & 1558 & 31 & 23 & $\gamma \mathrm{CC}(62)$ \\
\hline 21 & - & 1497 & 1604 & 1541 & 0 & 10 & $\gamma \mathrm{CC}(47)$ \\
\hline 22 & 1463 & - & 1507 & 1449 & 1 & 1 & $\beta \mathrm{HCH}(62)$ \\
\hline 23 & - & 1454 & 1503 & 1445 & 2 & 2 & $\beta \mathrm{HCC}(24)+\beta \mathrm{HCH}(38)$ \\
\hline 24 & - & - & 1500 & 1441 & 1 & 3 & $\beta \mathrm{HCH}(75)+\tau \mathrm{HCCC}(14)$ \\
\hline 25 & 1439 & - & 1494 & 1436 & 8 & 5 & $\beta \mathrm{HNC}(17)+\beta \mathrm{HCH}(26)$ \\
\hline 26 & - & - & 1493 & 1435 & 7 & 5 & $\beta \mathrm{HNC}(14)+\beta \mathrm{HCH}(39)$ \\
\hline 27 & - & 1431 & 1492 & 1434 & 8 & 4 & $\beta \mathrm{HNC}(12)$ \\
\hline 28 & - & 1425 & 1482 & 1424 & 4 & 2 & $\beta \mathrm{HCH}(63)+\tau \mathrm{HCCC}(13)$ \\
\hline 29 & 1417 & - & 1482 & 1424 & 1 & 8 & $\beta \mathrm{HCH}(62)$ \\
\hline 30 & - & 1395 & 1459 & 1402 & 0 & 0 & $\gamma \mathrm{CC}(11)+\beta \mathrm{HCC}(55)$ \\
\hline 31 & - & - & 1422 & 1366 & 2 & 3 & $\beta \mathrm{HCH}(85)$ \\
\hline 32 & 1363 & - & 1415 & 1360 & 28 & 1 & $\gamma \mathrm{NC}(31)+\beta \mathrm{HNC}(11)$ \\
\hline 33 & - & 1352 & 1412 & 1357 & 1 & 0 & $\beta \mathrm{HCH}(89)$ \\
\hline 34 & - & - & 1403 & 1348 & 0 & 4 & $\beta \mathrm{HNC}(69)$ \\
\hline 35 & 1314 & 1317 & 1383 & 1329 & 0 & 3 & $\tau \operatorname{HCCC}(66)$ \\
\hline 36 & 1284 & 1287 & 1338 & 1285 & 0 & 5 & $\beta \mathrm{HCC}(57)+\tau \mathrm{HCCC}(11)$ \\
\hline 37 & - & - & 1317 & 1266 & 0 & 0 & $\beta \mathrm{HCC}(10)+\tau \mathrm{HCCC}(37)$ \\
\hline 38 & 1263 & - & 1312 & 1261 & 1 & 2 & $\gamma \mathrm{CC}(74)$ \\
\hline 39 & - & - & 1301 & 1250 & 0 & 1 & $\gamma \mathrm{CC}(14)+\beta \mathrm{HCC}(55)$ \\
\hline 40 & 1230 & 1232 & 1295 & 1244 & 1 & 2 & $\beta \mathrm{HCC}(26)$ \\
\hline 41 & 1214 & 1211 & 1237 & 1189 & 1 & 7 & $\gamma \mathrm{NC}(10)+\beta \mathrm{HCC}(12)$ \\
\hline 42 & 1178 & 1180 & 1223 & 1176 & 0 & 11 & $\gamma \mathrm{CC}(56)+\beta \mathrm{HCC}(16)$ \\
\hline 43 & - & 1169 & 1208 & 1161 & 10 & 1 & $\gamma \mathrm{NC}(45)+\gamma \mathrm{CC}(29)$ \\
\hline 44 & 1155 & - & 1188 & 1142 & 0 & 2 & $\gamma \mathrm{CC}(10)+\beta \mathrm{HCC}(73)$ \\
\hline 45 & 1117 & 1123 & 1160 & 1115 & 3 & 2 & $\gamma \mathrm{NC}(46)$ \\
\hline 46 & - & 1094 & 1152 & 1107 & 0 & 2 & $\gamma \mathrm{CC}(33)+\beta \mathrm{HCC}(35)$ \\
\hline 47 & 1090 & - & 1108 & 1065 & 1 & 4 & $\gamma \mathrm{CC}(20)+\tau \mathrm{HCCC}(35)$ \\
\hline 48 & 1047 & - & 1098 & 1056 & 3 & 2 & $\gamma \mathrm{CC}(18)+\beta \mathrm{HNC}(11)$ \\
\hline 49 & - & 1033 & 1071 & 1029 & 0 & 3 & $\gamma \mathrm{CC}(17)+\beta \mathrm{HCC}(16)+\beta \mathrm{CCC}(16)$ \\
\hline 50 & 1027 & - & 1063 & 1021 & 1 & 0 & $\begin{array}{l}\beta \mathrm{HCH}(14)+\tau \mathrm{HCCC}(59)+ \\
\omega \mathrm{CCCC}(11)\end{array}$ \\
\hline 51 & 1010 & - & 1054 & 1013 & 2 & 16 & $\gamma \mathrm{CC}(31)+\gamma \mathrm{SC}(11)+\beta \mathrm{CCC}(29)$ \\
\hline 52 & - & 1003 & 1038 & 997 & 0 & 2 & $\gamma \mathrm{CC}(92)$ \\
\hline 53 & 984 & - & 1016 & 976 & 3 & 4 & $\gamma \mathrm{NC}(28)+\beta \mathrm{HNC}(11)+\beta \mathrm{CNC}(16)$ \\
\hline 54 & 971 & - & 1008 & 969 & 0 & 0 & $\tau \operatorname{HCCC}(65)+\tau \operatorname{CCCC}(10)$ \\
\hline
\end{tabular}




\begin{tabular}{|c|c|c|c|c|c|c|c|}
\hline 1 & 2 & 3 & 4 & 5 & 6 & 7 & 8 \\
\hline 55 & 958 & - & 1007 & 968 & 0 & 0 & $\beta \mathrm{HCH}(10)+\tau \mathrm{HCCC}(45)$ \\
\hline 56 & 921 & 924 & 972 & 934 & 0 & 0 & $\tau \mathrm{HCCC}(86)$ \\
\hline 57 & 894 & 895 & 908 & 873 & 2 & 5 & $\gamma \mathrm{NC}(15)+\gamma \mathrm{SC}(16)$ \\
\hline 58 & 880 & - & 897 & 862 & 0 & 4 & $\gamma \mathrm{CC}(38)+\tau \mathrm{HCCC}(13)$ \\
\hline 59 & - & 855 & 886 & 852 & 0 & 0 & $\tau \mathrm{HCCC}(86)$ \\
\hline 60 & 839 & 835 & 861 & 828 & 1 & 1 & $\tau \mathrm{HCCC}(14)$ \\
\hline 61 & 783 & 798 & 813 & 781 & 0 & 4 & $\gamma \mathrm{CC}(25)+\beta \mathrm{CCC}(47)$ \\
\hline 62 & 754 & - & 799 & 768 & 1 & 0 & $\begin{array}{l}\gamma \mathrm{CC}(13)+\beta \mathrm{NCN}(22)+\omega \\
\mathrm{ONCC}(11)\end{array}$ \\
\hline 63 & 735 & - & 772 & 742 & 5 & 0 & $\tau \operatorname{HCCC}(74)$ \\
\hline 64 & - & 728 & 763 & 733 & 1 & 0 & $\omega \mathrm{ONCC}(49)$ \\
\hline 65 & 725 & - & 750 & 721 & 5 & 0 & $\omega \mathrm{ONCC}(93)$ \\
\hline 66 & - & 708 & 742 & 713 & 1 & 0 & $\beta \mathrm{HCC}(18)+\tau \mathrm{HCCC}(28)$ \\
\hline 67 & 698 & - & 730 & 702 & 1 & 0 & $\tau \mathrm{CCCC}(58)$ \\
\hline 68 & 668 & 674 & 687 & 661 & 0 & 3 & $\gamma \mathrm{SC}(16)+\beta \mathrm{CCC}(54)$ \\
\hline 69 & 645 & 640 & 669 & 643 & 10 & 0 & $\tau \mathrm{HNCN}(70)+\omega \mathrm{CNSC}(12)$ \\
\hline 70 & 610 & 608 & 645 & 619 & 1 & 0 & $\beta \mathrm{OCN}(46)$ \\
\hline 71 & 590 & 589 & 588 & 565 & 1 & 1 & $\begin{array}{l}\beta \mathrm{CNC}(11)+\tau \mathrm{HNCN}(14)+\omega \\
\mathrm{ONCC}(10)\end{array}$ \\
\hline 72 & 568 & 553 & 586 & 563 & 0 & 3 & $\tau \mathrm{HNCN}(28)+\omega \mathrm{CNSC}(14)$ \\
\hline 73 & 555 & 531 & 559 & 537 & 4 & 0 & $\tau \mathrm{HNCN}(53)$ \\
\hline 74 & 535 & - & 559 & 537 & 0 & 4 & $\gamma \mathrm{CC}(17)+\beta \mathrm{CCC}(27)$ \\
\hline 75 & 505 & - & 531 & 510 & 1 & 1 & $\begin{array}{l}\tau \mathrm{CCCC}(14)+\omega \mathrm{CCCC}(10)+\omega \mathrm{SCCC} \\
(19)\end{array}$ \\
\hline 76 & 470 & 485 & 522 & 501 & 3 & 1 & $\begin{array}{l}\beta \mathrm{NCN}(10)+\beta \mathrm{CNC}(10)+\tau \\
\mathrm{HNCN}(14)+\omega \mathrm{CNSC}(15)\end{array}$ \\
\hline 77 & 459 & 449 & 470 & 452 & 1 & 0 & $\tau \mathrm{CCCC}(10)+\omega \mathrm{CCCC}(24)$ \\
\hline 78 & - & - & 449 & 432 & 0 & 1 & $\gamma \mathrm{SC}(21)+\beta \mathrm{CCC}(19)$ \\
\hline 79 & - & 396 & 434 & 418 & 4 & 1 & $\gamma \mathrm{SC}(21)+\beta \mathrm{OCN}(15)$ \\
\hline 80 & - & - & 388 & 373 & 0 & 0 & $\beta \mathrm{CCC}(28)+\beta \operatorname{SCC}(15)$ \\
\hline 81 & - & - & 376 & 361 & 1 & 0 & $\begin{array}{l}\beta \mathrm{CCC}(12)+\omega \operatorname{CCCC}(21)+\omega \\
\operatorname{CNSC}(10)\end{array}$ \\
\hline 82 & - & 331 & 370 & 355 & 1 & 1 & $\gamma \mathrm{SC}(12)+\beta \mathrm{OCN}(29)$ \\
\hline 83 & - & 302 & 324 & 311 & 0 & 1 & $\beta \operatorname{CCC}(38)$ \\
\hline 84 & - & - & 291 & 280 & 0 & 1 & $\gamma \mathrm{CC}(11)+\beta \operatorname{CCC}(41)$ \\
\hline 85 & - & 254 & 258 & 248 & 1 & 0 & $\tau \mathrm{CCCC}(10)+\omega \mathrm{CCCC}(17)$ \\
\hline 86 & - & - & 250 & 240 & 0 & 0 & $\beta \mathrm{CCC}(12)+\beta \mathrm{SCC}(46)$ \\
\hline 87 & - & - & 241 & 232 & 0 & 0 & $\tau \mathrm{HCCC}(69)$ \\
\hline 88 & - & 185 & 189 & 182 & 0 & 1 & $\beta \mathrm{NCS}(22)$ \\
\hline 89 & - & - & 166 & 160 & 0 & 0 & $\begin{array}{l}\beta \mathrm{CCC}(12)+\tau \mathrm{CCCC}(13)+\tau \\
\mathrm{NCNC}(13)+\tau \mathrm{CNCS}(10)+\omega \\
\mathrm{CCCC}(10)\end{array}$ \\
\hline 90 & - & - & 152 & 146 & 0 & 0 & $\tau \mathrm{CCCC}(20)+\tau \mathrm{CNCN}(32)$ \\
\hline 91 & - & 131 & 149 & 143 & 0 & 0 & $\begin{array}{l}\beta \mathrm{NCS}(10)+\beta \operatorname{CCC}(16)+\tau \\
\operatorname{CCCC}(12)+\tau \mathrm{CNCN}(14)+\omega \\
\operatorname{CCCC}(10)\end{array}$ \\
\hline 92 & - & - & 125 & 120 & 0 & 0 & $\begin{array}{l}\tau \mathrm{NCNC}(15)+\tau \mathrm{CNCS}(32)+\tau \\
\mathrm{CNCN}(21)\end{array}$ \\
\hline 93 & - & - & 119 & 115 & 0 & 0 & $\begin{array}{l}\tau \mathrm{HCCC}(52)+\tau \mathrm{CNCS}(12)+\omega \\
\operatorname{CCCC}(10)\end{array}$ \\
\hline 94 & - & - & 88 & 85 & 0 & 0 & $\tau \mathrm{HCCC}(10)+\tau \mathrm{CCCC}(73)$ \\
\hline 95 & - & - & 60 & 58 & 0 & 1 & $\begin{array}{l}\tau \mathrm{NCNC}(30)+\tau \\
\mathrm{CNCS}(20)+\tau \mathrm{CNCN}(14)+\omega \mathrm{CCCC}(1 \\
3)\end{array}$ \\
\hline 96 & - & - & 56 & 54 & 0 & 1 & $\beta \operatorname{CSC}(28)+\omega \operatorname{SCCC}(26)$ \\
\hline 97 & - & - & 42 & 41 & 0 & 1 & $\begin{array}{l}\beta \operatorname{CCC}(10)+\tau \operatorname{CCCC}(47)+ \\
\tau \operatorname{CSCC}(17)\end{array}$ \\
\hline 98 & - & - & 33 & 31 & 0 & 2 & $\tau \operatorname{CSCC}(64)$ \\
\hline 99 & & - & 17 & 16 & 0 & 0 & $\tau \operatorname{NCSC}(82)$ \\
\hline
\end{tabular}

${ }^{\mathrm{a}} \gamma$ : Stretching, $\gamma_{\mathrm{a}}$ : symmetrical stretching, $\gamma_{\mathrm{as}}$ : symmetrical stretching, $\beta$ : in-plane bending, $\omega$ : out-plane bending, $\tau$ : torsion

${ }^{\mathrm{b}}$ Scaling factor : 0.961 for B3LYP/6-311+G(d,p)

${ }^{\mathrm{c}}$ Relative absorption intensities normalized with the highest peak absorption equal to 100 .

${ }^{\mathrm{d}}$ Relative Raman intensities normalized to 100 . 
In aromatic compounds, the methyl asymmetric vibrations are expected near in the range $3000-2925 \mathrm{~cm}^{-1}$ and the symmetric $\mathrm{CH}_{3}$ vibrations in the range 2940-2905 [53, 54]. In the title compound, the asymmetric stretching vibration was identified at 2968, 2966, $2965 \mathrm{~cm}^{-1}$ in the B3LYP/6-311++G(d,p) method which showed good agreement with recorded FT-Raman spectrum at $2980,2962 \mathrm{~cm}^{-1}$, and were not detected in the in the FT-IR spectrum. The $\mathrm{CH}_{3}$ symmetric stretching was found at $2915,2901 \mathrm{~cm}^{-1}$ in the B3LYP/6-311++G(d,p) method and at 2912, 2870 $\mathrm{cm}^{-1}$ in FT-IR spectrum. The PED corresponding to asymmetric and symmetric stretching vibration is almost $85 \%$ and $97 \%$, as shown in Table 2.

For the assignments of $\mathrm{CH}_{2}$ group frequencies, basically, six fundamentals can be associated to each $\mathrm{CH}_{2}$ group which is expected to be depolarized [55]. The $\mathrm{CH}_{2}$ asymmetrical stretching vibration is generally observed above $3000 \mathrm{~cm}^{-1}$, while the symmetrical stretch would appear between 3000 and $2900 \mathrm{~cm}^{-1}$ [56]. In this study, the asymmetric stretching vibrations were observed at $3166,3075,3074 \mathrm{~cm}^{-1}$ in the B3LYP/6$311++\mathrm{G}(\mathrm{d}, \mathrm{p})$ method, $2961 \mathrm{~cm}^{-1}$ in the FT-IR spectrum and $2931 \mathrm{~cm}^{-1}$ in the FT-Raman spectrum. The symmetric $\mathrm{CH}_{2}$ stretching vibrations are identified at 2916, $2903 \mathrm{~cm}^{-1}$ in the B3LYP/6$311++\mathrm{G}(\mathrm{d}, \mathrm{p})$ method, $2929 \mathrm{~cm}^{-1}$ in the FR-IR spectrum and $2871 \mathrm{~cm}^{-1}$ in the FT-Raman spectrum.

\subsection{HOMO-LUMO energy}

The fundamental importance of the HOMO (highest occupied molecular orbital) and LUMO (lowest unoccupied molecular orbital) is understanding the chemical stability and reactivity of many organic molecules [57]. HOMO, the outermost orbital containing electrons, tends to give these electrons; on the other hand, LUMO, the innermost orbital, contains free places to accept electrons [58]. HOMO and LUMO are directly related to the ionization potential and electron affinity.

The energy difference between HOMO and LUMO orbits is called energy gap which is considered as important for indicator for compound stability [59]. The total energy, HOMO and LUMO energies, the energy gap $(\Delta \mathrm{E})$, the ionization potential (I), the electron affinity (A), the absolute electronegativity $(\chi)$, the absolute hardness $(\eta)$ and softness (S) of the title molecule have been calculated at the B3LYP/6-311++G(d, P) basis set (Fig. 4); the results are shown in Table 3.

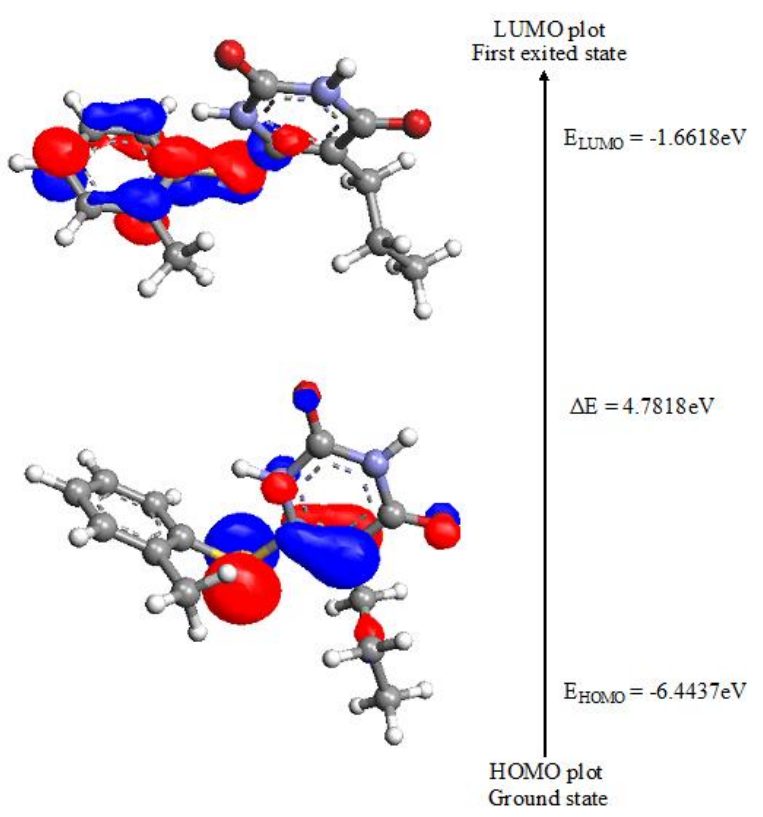

Fig. 4. Atomic orbital HOMO-LUMO composition of the frontier molecular orbital of the title compound

Table 3

Comparison of the HOMO, LUMO, energy gaps and ionization potentials of the title compound

\begin{tabular}{lc}
\hline \hline Basis set & B3LYP/6-311++G(d, p) \\
\hline $\mathbf{E}_{\text {Homo }}$ & -6.4437 \\
$\mathbf{E}_{\text {Lomo }}$ & -1.6618 \\
Ionization potential & 6.4437 \\
Electron affinity & 1.6618 \\
Energy gap & 4.7818 \\
Electronegativity & 4.0527 \\
Chemical potential & 4.0527 \\
Chemical hardness & 2.3909 \\
Chemical softness & 0.2091 \\
Electrophilicity index & 3.4347 \\
\hline \hline
\end{tabular}

\subsection{Local reactivity descriptors}

The local quantities such as Fukui function and local softness define the reactivity/selectivity of a specific site in a molecule. The Fukui function is defined as follows:

$$
f(\vec{r})=(\partial \rho / \partial \mathrm{N}) v(\vec{r})=\left(\frac{\delta \mu}{\delta v(\vec{r})}\right) N .
$$

Kolandaival et al. [60] introduced the atomic descriptor to determine the local reactive sites of the molecular system. The individual atomic charges calculated by Mulliken population analysis (MPA) have been used to calculated the Fukui function. 
Table 4

Condensed Fukui function $f_{r}$ and new descriptor $(s f)_{r}$ of the title compound

\begin{tabular}{|c|c|c|c|c|c|c|}
\hline $\begin{array}{l}\text { Atoms } \\
\end{array}$ & $\overline{f_{\mathrm{r}}^{+}}$ & $\overline{f_{\mathrm{r}}^{-}}$ & $\overline{f_{\mathrm{r}}{ }^{0}}$ & 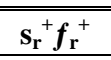 & $\overline{\mathbf{s}_{\mathrm{r}}^{-} \boldsymbol{f}_{\mathrm{r}}^{-}}$ & $\overline{s r^{0} f r^{0}}$ \\
\hline C1 & 0.0016 & 0.0015 & 0.0015 & 0.0003 & 0.0003 & 0.0003 \\
\hline N2 & -0.0010 & -0.0089 & -0.0049 & 0.0002 & 0.0018 & 0.0010 \\
\hline $\mathrm{C} 3$ & -0.0231 & -0.0087 & -0.0159 & 0.0048 & 0.0018 & 0.0033 \\
\hline $\mathrm{C} 4$ & -0.0314 & -0.0461 & -0.0388 & 0.0065 & 0.0096 & 0.0081 \\
\hline $\mathrm{C} 4$ & 0.0310 & -0.0091 & 0.0109 & 0.0064 & 0.0019 & 0.0022 \\
\hline N6 & -0.0371 & -0.0509 & -0.0440 & 0.0077 & 0.0106 & 0.0092 \\
\hline O7 & -0.0559 & -0.1110 & -0.0835 & 0.0117 & 0.0232 & 0.0174 \\
\hline 08 & -0.1010 & -0.0971 & -0.0991 & 0.0211 & 0.0203 & 0.0207 \\
\hline C9 & 0.0097 & 0.0086 & 0.0092 & 0.0020 & 0.0018 & 0.0019 \\
\hline C10 & -0.0081 & 0.0179 & 0.0049 & 0.0017 & 0.0037 & 0.0010 \\
\hline C11 & -0.0071 & -0.0390 & -0.0230 & 0.0014 & 0.0081 & 0.0048 \\
\hline S12 & -0.1761 & -0.1814 & -0.1787 & 0.0368 & 0.0379 & 0.0373 \\
\hline C13 & 0.5241 & 0.1789 & 0.3515 & 0.1096 & 0.0374 & 0.0735 \\
\hline C14 & -0.0787 & -0.0297 & -0.0542 & 0.0164 & 0.0062 & 0.0113 \\
\hline C15 & -0.1221 & -0.0333 & -0.0777 & 0.0255 & 0.0069 & 0.0162 \\
\hline C16 & -0.0191 & -0.0163 & -0.0177 & 0.0040 & 0.0034 & 0.0037 \\
\hline C17 & -0.0643 & -0.0473 & -0.0558 & 0.0134 & 0.0099 & 0.0116 \\
\hline C18 & -0.1715 & -0.0768 & -0.1242 & 0.0358 & 0.0160 & 0.0259 \\
\hline C19 & -0.0288 & -0.0197 & -0.0242 & 0.0060 & 0.0041 & 0.0050 \\
\hline H2O & -0.0417 & -0.0372 & -0.0394 & 0.0087 & 0.0077 & 0.0082 \\
\hline H21 & -0.0028 & -0.0243 & -0.0136 & 0.0006 & 0.0050 & 0.0028 \\
\hline H22 & -0.0626 & -0.0530 & -0.0578 & 0.0131 & 0.0111 & 0.0121 \\
\hline H23 & -0.0249 & -0.0173 & -0.0211 & 0.0052 & 0.0036 & 0.0044 \\
\hline H24 & -0.0120 & -0.0123 & -0.0122 & 0.0025 & 0.0025 & 0.0025 \\
\hline H25 & 0.0028 & -0.0153 & -0.0062 & 0.0006 & 0.0032 & 0.0013 \\
\hline H26 & -0.0264 & -0.0327 & -0.0296 & 0.0055 & 0.0068 & 0.0061 \\
\hline H27 & -0.0189 & -0.0208 & -0.0198 & 0.0039 & 0.0043 & 0.0041 \\
\hline H28 & -0.0070 & -0.0124 & -0.0097 & 0.0014 & 0.0026 & 0.0020 \\
\hline H29 & -0.0549 & -0.0406 & -0.0478 & 0.0114 & 0.0085 & 0.0100 \\
\hline H30 & -0.0593 & -0.0462 & -0.0527 & 0.0124 & 0.0096 & 0.0110 \\
\hline H31 & -0.0501 & -0.0365 & -0.0433 & 0.0104 & 0.0076 & 0.0090 \\
\hline H32 & -0.1687 & -0.0215 & -0.0951 & 0.0352 & 0.0045 & 0.0198 \\
\hline H33 & -0.0384 & -0.0300 & -0.0342 & 0.0080 & 0.0062 & 0.0071 \\
\hline H34 & -0.0444 & 0.0071 & -0.0186 & 0.0093 & 0.0014 & 0.0039 \\
\hline H35 & -0.0306 & -0.0376 & -0.0341 & 0.0064 & 0.0078 & 0.0071 \\
\hline
\end{tabular}

The Fukui function $\left(f^{+}(\vec{r}), f^{-}(\vec{r}), f^{0}(\vec{r})\right)$, $\left(s_{r}^{+}, s_{r}^{-}, s_{r}^{0}\right)[61]$ for selected atomic sites in the title molecule have been listed in Table 4. Yang and Mortier [62] have given a simple procedure for the calculation of the atomic condensed Fukui function indices based on the MPA and the three possible forward, backward, and central finite difference approximations to the derivatives [63]. The Fukui functions are calculated using the following equation:

$$
\begin{aligned}
& f^{+}(\vec{r})=q_{r}(N+1)-q_{r}(N) \\
& \quad \text { for nucleophilic attack } \\
& f^{-}(\vec{r})=q_{r}(N)-q_{r}(N-1) \\
& \quad \text { for electrophilic attack }
\end{aligned}
$$

$$
\begin{gathered}
f^{0}(\vec{r})=\left(q_{r}(N+1)-q_{r}(N-1)\right) / 2 \\
\quad \text { for radical attack }
\end{gathered}
$$

In these equations, the $q_{\mathrm{r}}$ is the atomic charge (evaluated from the Mulliken population analysis, electrostatic derived charge, etc.) at the $r^{\text {th }}$ atomic site is the neutral $(N)$, anionic $(N+1)$, cationic $(N-1)$ chemical species.

The local softness is related to Fukui function as follows:

$$
\begin{aligned}
\mathrm{s}(\vec{r})=\left(\frac{\partial \rho(\vec{r})}{\delta \mu}\right) v(\vec{r}) & =\left(\frac{\partial \rho(\vec{r})}{\partial N}\right) v(\vec{r})\left(\frac{\partial N)}{\partial \mu}\right) v(\vec{r}) \\
& =f(\vec{r}) \mathrm{S}
\end{aligned}
$$

where $\mathrm{S}$ is the global softness given as 


$$
s=\left(\frac{1}{2 \eta}\right)=\int s(\vec{r}) d \vec{r}
$$

The local softness can be represented as:

$$
\begin{gathered}
s_{r}^{+} f_{r}^{+}=f_{r}^{+} S \text { for nucleophilic attack } \\
s_{r}^{-} f_{r}^{-}=f_{r}^{-} S \text { for electrophilic attack } \\
s_{r}^{0} f_{r}^{0}=f_{r}^{0} S \text { for radical attack }
\end{gathered}
$$

where,,+- 0 signs show nucleophilic, electrophilic and radical attack, respectively.

Table 4 shows the $f_{\mathrm{r}}$ and $\left(s_{\mathrm{r}} f_{\mathrm{r}}\right)$ values for the title compound, using which one can find the complexities associated with $f_{\mathrm{r}}$ values due to the negative values being removed in the $\left(s_{\mathrm{r}} f_{\mathrm{r}}\right)$ values. It has been found that the MAP schemes predicting $\mathrm{C}_{13}$ to have a higher $f_{r}^{+}$value indicate possible sites for nucleophilic attack. From the values reported in Table 4, the MAP schemes predict the reactivity order for the nucleophilic case to be $\mathrm{C}_{13}>\mathrm{C}_{5}>\mathrm{C}_{9}$ $>\mathrm{H}_{28}>\mathrm{C}_{1}$. The observation of the reactive sites by $s_{r}^{+} f_{r}^{+}$is found almost identical to $f_{r}^{+}$. Even though the $\left(s_{\mathrm{r}} f_{\mathrm{r}}\right)$ values are numerically lower, it is worth nothing that the values are positive and the ordering of the reactivity has not changed in any case.

The calculated $\mathrm{f}_{\mathrm{r}}^{-}$values predicts that the possible sites for electrophilic attack are $\mathrm{C}_{13}>\mathrm{C}_{10}>\mathrm{C}_{9}>$ $\mathrm{H}_{34}>\mathrm{C}_{1}$ and the radical attack was predicted at $\mathrm{C}_{13}$ $>\mathrm{C}_{5}>\mathrm{C}_{9}>\mathrm{C}_{10}>\mathrm{C}_{1}$ site. It could therefore be concluded that the possibility of nucleophilic attack is higher than that of electrophilic and radical attack.

\subsection{Natural bond orbital (NBO) analysis}

In order to investigate the intra- and intermolecular interactions, the stabilization energies of the title compound were performed by using the second-order perturbation theory. The change in electron density (ED) in the $\left(\sigma^{* *}\right)$ anti-bonding orbitals and $E(2)$ energies have been calculated by the natural bond orbital analysis [64]. The second order Fock matrix was carried out to evaluate the donor-acceptor interactions in the NBO analysis [65]. The NBO analysis provides the most accurate possible 'natural Lewis structure' picture of $\phi$ because all of the orbital details are mathematically chosen to include the highest possible percentage of electron density.

The natural bond orbital analysis provides an efficient method for studying intra- and inter- molecular bonding and interaction among bonds and also provides a convenient basis for investigating charge transfer or conjugative interaction in molecular systems. Some electron donor orbital, acceptor orbital and interacting stabilization energy resulting from the second-order micro-disturbance theory have been reported [66]. For each donor (i) and acceptor (j), the stabilization energy E [2] associated with the delocalization $\mathrm{i}, \mathrm{j}$ estimated as:

$$
E_{2}=\Delta E_{\mathrm{ij}}=q_{\mathrm{i}} \frac{F(\mathrm{i}, \mathrm{j})^{2}}{E_{\mathrm{i}}-E_{\mathrm{j}}}
$$

where $q_{\mathrm{i}}$ is the donor orbital occupancy, $E_{\mathrm{i}}$ and $E_{\mathrm{j}}$ are diagonal elements and $F(\mathrm{i}, \mathrm{j})$ is the off-diagonal NBO Fock matrix elements.

The larger the $E(2)$ value, the more intensive the interaction between electron donors and electron acceptors is; i.e. the high donating tendency from electron donors to electron acceptors and the greater the extent of conjugation of the whole system. The delocalization of electron density between the occupied Lewis-type (bond or lone pair) NBO orbitals and formally unoccupied (anti-bond or Rydgberg) non-Lewis NBO orbitals correspond to a stabilizing donor-acceptor interaction. The $\mathrm{NBO}$ analysis has been performed on the title molecule at the B3LYP/6-311++G(d,p) level in order to determine the delocalization of electron density within the molecule.

The strong intramolecular hyperconjugative interaction of the $s$ and $p$ electrons of C-C to the anti $\mathrm{C}-\mathrm{C}$ bond of the ring leads to stabilization of some part of the ring, as shown in Table 5. The intramolecular hyperconjugative interaction of $\sigma$ $\left(\mathrm{C}_{4}-\mathrm{C}_{5}\right)$ distributes to $\sigma^{*}\left(\mathrm{C}_{3}-\mathrm{C}_{4}\right),\left(\mathrm{C}_{1}-\mathrm{N}_{2}\right),\left(\mathrm{C}_{1}-\mathrm{N}_{6}\right)$ leading to stabilization of $8.32 \mathrm{KJ} / \mathrm{mol}$. This enhances further conjugation with anti-bonding orbital of $\pi^{*}\left(\mathrm{C}_{3}-\mathrm{O}_{8}\right),\left(\mathrm{C}_{17}-\mathrm{C}_{18}\right)$ which lead to strong delocalization of $20.67 \mathrm{KJ} / \mathrm{mol}$, respectively. The $\sigma$ system shows some contribution to the delocalization corresponds to the donor-acceptor interactions are $\left(\mathrm{N}_{2}-\mathrm{H}_{20}\right) \rightarrow\left(\mathrm{C}_{5}-\mathrm{S}_{12}\right),\left(\mathrm{C}_{5}-\mathrm{S}_{12}\right) \rightarrow\left(\mathrm{C}_{1}-\mathrm{C}_{2}\right),\left(\mathrm{C}_{1}\right.$ $\left.\mathrm{O}_{7}\right) \rightarrow\left(\mathrm{C}_{1}-\mathrm{N}_{6}\right),\left(\mathrm{C}_{5}-\mathrm{N}_{6}\right) \rightarrow\left(\mathrm{C}_{4}-\mathrm{C}_{5}\right),\left(\mathrm{C}_{4}-\mathrm{C}_{9}\right) \rightarrow\left(\mathrm{C}_{1}-\right.$ $\left.\mathrm{N}_{6}\right),\left(\mathrm{C}_{3}-\mathrm{C}_{4}\right) \rightarrow\left(\mathrm{N}_{2}-\mathrm{H}_{20}\right)$ bondings are shown in Table 5. The LP (1) $\mathrm{N}_{6}$ anti-bonding acceptor $\pi^{*}$ $\left(\mathrm{C}_{1}-\mathrm{O}_{7}\right)$ of the title compound energy of 108.49 $\mathrm{KJ} / \mathrm{mol}$. The LP (1) $\mathrm{N}_{2}$ of the NBO orbital further conjugate with $\pi^{*}\left(\mathrm{C}_{1}-\mathrm{O}_{7}\right)$ resulting in an enormous stabilization of $108.49 \mathrm{KJ} / \mathrm{mol}$, as shown in Table 5 . This is the highest energy from all interactions. 
T a ble 5

Second order perturbation theory analysis of Fock matrix in NBO basis of the title compound

\begin{tabular}{|c|c|c|c|c|c|c|c|c|}
\hline Donor (i) & Type & ED/e & Acceptor (i) & Type & ED/e & $\mathrm{E}(2)^{\mathrm{a}}\left(\mathrm{kjmol}^{-1}\right)$ & $\mathbf{E}(\mathbf{j})-\mathbf{E}(\mathbf{i})^{\mathbf{b}}\left(\mathbf{a} . \mathbf{u}_{\mathbf{c}}\right)$ & $\mathbf{F}(\mathbf{i}, \mathbf{j})^{\mathbf{c}}(\mathbf{a} . \mathbf{u})$. \\
\hline \multirow[t]{2}{*}{$\mathrm{C}_{1}-\mathrm{N}_{2}$} & $\sigma$ & 1.9818 & $\mathrm{C}_{1}-\mathrm{O}_{7}$ & $\sigma^{*}$ & 0.0172 & 4.23 & 1.66 & 0.075 \\
\hline & $\sigma$ & & $\mathrm{N}_{2}-\mathrm{C}_{3}$ & $\sigma^{*}$ & 0.0872 & 4.05 & 1.47 & 0.070 \\
\hline \multirow[t]{2}{*}{$\mathrm{C}_{1}-\mathrm{N}_{6}$} & $\sigma$ & 1.9759 & $\mathrm{~N}_{2}-\mathrm{H}_{20}$ & $\sigma^{*}$ & 0.0244 & 3.33 & 1.19 & 0.056 \\
\hline & $\sigma$ & & $\mathrm{C}_{5}-\mathrm{S}_{12}$ & $\sigma^{*}$ & 0.0488 & 3.99 & 0.99 & 0.056 \\
\hline $\mathrm{C}_{1}-\mathrm{O}_{7}$ & $\sigma$ & 1.9843 & $\mathrm{C}_{1}-\mathrm{N}_{2}$ & $\sigma^{*}$ & 0.0498 & 5.20 & 1.78 & 0.087 \\
\hline $\mathrm{C}_{1}-\mathrm{O}_{7}$ & $\pi$ & 1.9868 & $\mathrm{C}_{1}-\mathrm{O}_{7}$ & $\pi^{*}$ & 0.4167 & 3.77 & 0.38 & 0.038 \\
\hline \multirow[t]{2}{*}{$\mathbf{N}_{2}-C_{3}$} & $\sigma$ & 1.9833 & $\mathrm{C}_{1}-\mathrm{N}_{2}$ & $\sigma^{*}$ & 0.0498 & 4.34 & 1.59 & 0.075 \\
\hline & $\sigma$ & & $\mathrm{C}_{1}-\mathrm{O}_{7}$ & $\sigma^{*}$ & 0.0172 & 3.61 & 1.49 & 0.066 \\
\hline $\mathbf{N}_{2}-H_{20}$ & $\sigma$ & 1.9726 & $\mathrm{C}_{1}-\mathrm{N}_{6}$ & $\sigma^{*}$ & 0.0876 & 8.32 & 1.11 & 0.087 \\
\hline $\mathrm{C}_{3}-\mathrm{C}_{4}$ & $\sigma$ & 1.9726 & $\mathrm{~N}_{2}-\mathrm{H}_{20}$ & $\sigma^{*}$ & 0.0244 & 3.14 & 1.00 & 0.050 \\
\hline $\mathrm{C}_{3}-\mathrm{O}_{8}$ & $\sigma$ & 1.9934 & $\mathrm{C}_{3}-\mathrm{C}_{4}$ & $\sigma^{*}$ & 0.0770 & 2.05 & 1.48 & 0.050 \\
\hline $\mathrm{C}_{3}-\mathrm{O}_{8}$ & $\pi$ & 1.9827 & $\mathrm{C}_{4}-\mathrm{C}_{5}$ & $\pi^{*}$ & 0.2423 & 4.13 & 0.41 & 0.039 \\
\hline \multirow{2}{*}{$\mathrm{C}_{4}-\mathrm{C}_{5}$} & $\sigma$ & 1.9754 & $\mathrm{C}_{4}-\mathrm{C}_{9}$ & $\sigma^{*}$ & 0.0187 & 4.07 & 1.22 & 0.063 \\
\hline & $\sigma$ & & $\mathrm{N}_{6}-\mathrm{H}_{21}$ & $\sigma^{*}$ & 0.0325 & 3.24 & 1.17 & 0.055 \\
\hline $\mathrm{C}_{4}-\mathrm{C}_{5}$ & $\sigma$ & 1.8445 & $\mathrm{C}_{3}-\mathrm{O}_{8}$ & $\pi^{*}$ & 0.2952 & 17.63 & 0.31 & 0.068 \\
\hline \multirow{2}{*}{$\mathrm{C}_{4}-\mathrm{C}_{9}$} & $\sigma$ & 1.9714 & $\mathrm{C}_{4}-\mathrm{C}_{5}$ & $\sigma^{*}$ & 0.0260 & 4.79 & 1.27 & 0.070 \\
\hline & $\sigma$ & & $\mathrm{C}_{5}-\mathrm{N}_{6}$ & $\sigma^{*}$ & 0.0375 & 4.37 & 1.10 & 0.062 \\
\hline $\mathrm{C}_{5}-\mathrm{N}_{6}$ & $\sigma$ & 1.9852 & $\mathrm{C}_{4}-\mathrm{C}_{9}$ & $\sigma^{*}$ & 0.0187 & 3.01 & 1.31 & 0.056 \\
\hline \multirow{2}{*}{$\mathrm{C}_{5}-\mathrm{S}_{12}$} & $\sigma$ & 1.9655 & $\mathrm{C}_{1}-\mathrm{N}_{6}$ & $\sigma^{*}$ & 0.0876 & 3.22 & 1.07 & 0.053 \\
\hline & $\sigma$ & & $\mathrm{C}_{3}-\mathrm{C}_{4}$ & $\sigma^{*}$ & 0.0770 & 4.58 & 1.03 & 0.062 \\
\hline $\mathrm{N}_{6}-\mathrm{H}_{21}$ & $\sigma$ & 1.9806 & $\mathrm{C}_{4}-\mathrm{C}_{5}$ & $\sigma^{*}$ & 0.0260 & 3.39 & 1.33 & 0.060 \\
\hline $\mathrm{C}_{9}-\mathrm{H}_{22}$ & $\sigma$ & 1.9679 & $\mathrm{C}_{4}-\mathrm{C}_{5}$ & $\pi^{*}$ & 0.0242 & 4.29 & 0.50 & 0.440 \\
\hline $\mathrm{C}_{9}-\mathrm{H}_{23}$ & $\sigma$ & 1.9746 & $\mathrm{C}_{3}-\mathrm{C}_{4}$ & $\sigma^{*}$ & 0.0770 & 4.76 & 0.88 & 0.059 \\
\hline$S_{12}-C_{13}$ & $\sigma$ & 1.9687 & $\mathrm{C}_{14}-\mathrm{C}_{15}$ & $\sigma^{*}$ & 0.0236 & 3.64 & 1.17 & 0.058 \\
\hline $\mathrm{C}_{13}-\mathrm{C}_{14}$ & $\sigma$ & 1.9736 & $\mathrm{C}_{13}-\mathrm{C}_{18}$ & $\sigma^{*}$ & 0.0260 & 3.33 & 1.24 & 0.057 \\
\hline \multirow[t]{2}{*}{$\mathrm{C}_{13}-\mathrm{C}_{14}$} & $\pi$ & 1.6682 & $\mathrm{C}_{15}-\mathrm{C}_{16}$ & $\pi^{*}$ & 0.3149 & 16.17 & 0.28 & 0.610 \\
\hline & $\pi$ & & $\mathrm{C}_{17}-\mathrm{C}_{18}$ & $\pi^{*}$ & 0.0327 & 20.67 & 0.28 & 0.680 \\
\hline \multirow[t]{2}{*}{$\mathrm{C}_{14}-\mathrm{C}_{15}$} & $\sigma$ & 1.9705 & $\mathrm{~S}_{12}-\mathrm{C}_{13}$ & $\sigma^{*}$ & 0.0364 & 4.45 & 0.08 & 0.055 \\
\hline & $\sigma$ & & $\mathrm{C}_{13}-\mathrm{C}_{14}$ & $\sigma^{*}$ & 0.0359 & 3.90 & 1.23 & 0.062 \\
\hline \multirow[t]{2}{*}{$\mathrm{C}_{15}-\mathrm{C}_{16}$} & $\pi$ & 1.6650 & $\mathrm{C}_{13}-\mathrm{C}_{14}$ & $\pi^{*}$ & 0.3877 & 23.20 & 0.27 & 0.071 \\
\hline & $\pi$ & & $\mathrm{C}_{17}-\mathrm{C}_{18}$ & $\pi^{*}$ & 0.3277 & 19.39 & 0.27 & 0.064 \\
\hline $\mathrm{C}_{15}-\mathrm{H}_{29}$ & $\sigma$ & 1.9804 & $\mathrm{C}_{13}-\mathrm{C}_{14}$ & $\sigma^{*}$ & 0.0359 & 4.39 & 1.06 & 0.061 \\
\hline $\mathrm{C}_{17}-\mathrm{C}_{18}$ & $\sigma$ & 1.9755 & $\mathrm{~S}_{12}-\mathrm{C}_{13}$ & $\sigma^{*}$ & 0.0364 & 4.40 & 0.86 & 0.550 \\
\hline \multirow[t]{2}{*}{$\mathrm{C}_{17}-\mathrm{C}_{18}$} & $\pi$ & 1.6770 & $\mathrm{C}_{13}-\mathrm{C}_{14}$ & $\pi^{*}$ & 0.3877 & 17.47 & 0.27 & 0.063 \\
\hline & $\pi$ & & $\mathrm{C}_{15}-\mathrm{C}_{16}$ & $\pi^{*}$ & 0.3149 & 19.30 & 0.28 & 0.065 \\
\hline $\mathrm{C}_{18}-\mathrm{H}_{32}$ & $\sigma$ & 1.9682 & $\mathrm{~N}_{6}-\mathrm{H}_{21}$ & $\sigma^{*}$ & 0.0325 & 6.18 & 0.94 & 0.068 \\
\hline $\mathrm{C}_{19}-\mathrm{H}_{34}$ & $\sigma$ & 1.9855 & $\mathrm{C}_{14}-\mathrm{C}_{15}$ & $\sigma^{*}$ & 0.0260 & 4.75 & 1.04 & 0.063 \\
\hline $\mathrm{C}_{19}-\mathrm{H}_{35}$ & $\sigma$ & 1.9761 & $\mathrm{C}_{13}-\mathrm{C}_{14}$ & $\pi^{*}$ & 0.3877 & 4.01 & 0.51 & 0.045 \\
\hline \multirow[t]{2}{*}{$\mathbf{N}_{2}$} & LP (1) & 1.5884 & $\mathrm{C}_{1}-\mathrm{O}_{7}$ & $\pi^{*}$ & 0.4167 & 108.49 & 0.31 & 0.163 \\
\hline & & & $\mathrm{C}_{3}-\mathrm{O}_{8}$ & $\pi^{*}$ & 0.2952 & 51.76 & 0.30 & 0.115 \\
\hline \multirow[t]{2}{*}{$\mathbf{N}_{6}$} & LP (1) & 1.6576 & $\mathrm{C}_{1}-\mathrm{O}_{7}$ & $\pi^{*}$ & 0.4167 & 54.27 & 0.30 & 0.115 \\
\hline & LP (1) & & $\mathrm{C}_{4}-\mathrm{C}_{5}$ & $\pi^{*}$ & 0.2423 & 42.62 & 0.31 & 0.105 \\
\hline \multirow[t]{2}{*}{$\mathbf{O}_{7}$} & LP (2) & 1.8731 & $\mathrm{C}_{1}-\mathrm{N}_{2}$ & $\sigma^{*}$ & 0.0498 & 18.50 & 0.98 & 0.122 \\
\hline & LP (2) & & $\mathrm{C}_{1}-\mathrm{N}_{6}$ & $\sigma^{*}$ & 0.0876 & 25.56 & 0.68 & 0.199 \\
\hline \multirow[t]{2}{*}{$\mathrm{O}_{8}$} & LP (2) & 1.8609 & $\mathrm{~N}_{2}-\mathrm{C}_{3}$ & $\sigma^{*}$ & 0.0872 & 28.08 & 0.07 & 0.013 \\
\hline & LP (2) & & $\mathrm{C}_{3}-\mathrm{C}_{4}$ & $\sigma^{*}$ & 0.0770 & 19.99 & 0.66 & 0.104 \\
\hline$S_{12}$ & LP (2) & 1.8723 & $\mathrm{C}_{4}-\mathrm{C}_{5}$ & $\pi^{*}$ & 0.2423 & 6.33 & 0.28 & 0.039 \\
\hline $\mathrm{C}_{3}-\mathrm{O}_{8}$ & $\pi^{*}$ & 0.2952 & $\mathrm{C}_{4}-\mathrm{C}_{5}$ & $\pi^{*}$ & 0.2423 & 92.15 & 0.02 & 0.071 \\
\hline$C_{13}-C_{14}$ & $\pi^{*}$ & 0.3877 & $\mathrm{C}_{4}-\mathrm{C}_{5}$ & $\pi^{*}$ & 0.2423 & 5.73 & 0.01 & 0.015 \\
\hline
\end{tabular}

${ }^{a} E(2)$ : Energy of hyperconjugative interaction (stabilization energy).

${ }^{\mathrm{b}}$ Energy difference between donor and acceptor I and j NBO orbitals.

${ }^{\mathrm{c}} F(i . j)$ : The fork matrix element between I and j NBO orbitals. 


\subsection{Hyperpolarizability calculations}

The first order hyperpolarizability $\left(\beta_{\text {total }}\right)$ of the title compound along with the related properties $(\mu, \alpha$ and $\Delta \alpha)$ are calculated by using the DFTB3LYP method with $6-311++\mathrm{G}(\mathrm{d}, \mathrm{P})$ basis set based on the finite-field approach. The NLO effects arise from the interactions of electromagnetic fields in various media to produce new fields altered in phase, frequency, amplitude or other propagation characteristics from the incident fields. NLO is at the forefront of current research because of its importance in providing the key functions of frequency shifting, optical modulation, optical switching, optical logic, and optical memory for emerging technologies in areas such as telecommunications, signal processing, and optical interconnections [67].

The non-linear optical response of an isolated molecule in an electric field $E_{\mathrm{i}}(\omega)$ can be represented as a Taylor series expansion of the total dipole moment, $\mu_{\text {tot }}$, induced by the field:

$$
\mu_{\mathrm{tot}}=\mu_{0}+\alpha_{\mathrm{ij}} E_{\mathrm{j}}+B_{\mathrm{ijk}} E_{\mathrm{j}} E_{\mathrm{k}}+\ldots,
$$

where $\alpha$ is the linear polarizability, $\mu_{0}$ is the permanent dipole moment and $\beta_{\mathrm{ijk}}$ are the first hyperpolarizability tensor components. The isotropic (or average) linear polarizability is defined as:

$$
\alpha=\frac{\alpha_{x x}+\alpha_{y y}+\alpha_{z z}}{3} .
$$

The first hyperpolarizability is a third rank tensor that can be described by $3 \times 3 \times 3$ matrix. The 27 components of 3D matrix can be reduced to 10 components due to the Kleinman symmetry [68].
Components of the first hyperpolarizability can be calculated using the following equation:

$$
\beta_{i}=\beta_{i i i}+\sum_{i \neq j}\left(\beta_{i j j}+\beta_{j i j}+\beta_{j j i}\right)
$$

Using the $x, y$ and $z$ components of $\beta$, the magnitude of the first hyperpolarizability tensor can be calculated by:

$$
\beta_{t o t}=\sqrt{\left(\beta_{x}^{2}+\beta_{y}^{2}+\beta_{z}^{2}\right)}
$$

The complete equation for calculating the magnitude of $\beta$ from Gaussian 03W output is given as follows:

$$
\begin{aligned}
& \beta_{\text {tot }}= \\
& =\sqrt{\begin{array}{c}
\left(\beta_{x x x}+\beta_{x y y}+\beta_{x z z}\right)^{2}+\left(\beta_{y y y}+\beta_{y z z}+\beta_{x x y}\right)^{2}+ \\
+\left(\beta_{z z z}+\beta_{x x z}+\beta_{y y z}\right)^{2}
\end{array}}
\end{aligned}
$$

The calculations of the total molecular dipole moment $(\mu)$, linear polarizability $(\alpha)$ and firstorder hyperpolarizability $(\beta)$ from the Gaussian output have been previously explained in detail [69], and the DFT has been extensively used as an effective method to investigate the organic NLO materials [70,71]. In addition, the polar properties of the title compound were calculated at the DFT (B3LYP)/6-311++G(d,p) level using Gaussian $03 \mathrm{~W}$ program package.

Urea is one of the prototypical molecules used in the study of the NLO properties of the molecules. Therefore, it was frequently used as a threshold value for comparative purposes.

Table 6

The values of calculated dipole moment $\mu_{(D)}$, polarizability $\left(\alpha_{0}\right)$, the first order hyperpolarizability $\left(\beta_{\text {tot }}\right)$ components of the title compound

\begin{tabular}{cccc}
\hline \hline Parameters & B3LYP/6-311++G(d,p) & Parameters & B3LYP/6-311++G(d,p) \\
\hline$\mu_{x}$ & 0.1418 & $\beta_{x x x}$ & 87.4914 \\
$\mu_{y}$ & 1.4189 & $\beta x x y$ & 209.2697 \\
$\mu_{z}$ & 0.8954 & $\beta x y y$ & 38.0065 \\
$\mu_{(D)}$ & 1.6837 & $\beta y y y$ & -169.2489 \\
$\alpha_{x x}$ & 246.9805 & $\beta z x x$ & 38.1262 \\
$\alpha_{x y}$ & 1.2806 & $\beta x y z$ & -1.042 \\
$\alpha_{y y}$ & 191.87 & $\beta z y y$ & -41.707 \\
$\alpha_{x z}$ & 19.83073 & $\beta x z z$ & 20.3421 \\
$\alpha_{y z}$ & 31.6945 & $\beta y z z$ & 78.9891 \\
$\alpha_{z z}$ & 186.4042 & $\beta z z z$ & 21.3964 \\
$\alpha_{0}($ e.s.u) & $3.0888 \times 10^{-23}$ & $\beta_{\text {tot }}($ e.s.u) & $1.6335 \times 10^{-30}$ \\
$\Delta \alpha$ (e.s.u) & $6.3978 \times 10^{-23}$ & & \\
\hline \hline
\end{tabular}


The calculated dipole moment and hyperpolarizability values obtained from B3LYP/6$31++\mathrm{G}(\mathrm{d}, \mathrm{p})$ methods are shown in Table 6. The total molecular dipole moment of the title molecule from the B3LYP with $6-311++\mathrm{G}(\mathrm{d}, \mathrm{p})$ basis set is 1.2 times greater than that of urea $(\mu=1.3732 \mathrm{D})$. Similarly, the first order hyperpolarizability of the title molecule with B3LYP/6-311++G(d,p) basis set $\left(1.6335 \times 10^{-30}\right)$ is four times greater than the value of urea $\left(\beta_{0}=0.372 \times 10^{-30} \mathrm{esu}\right)$. From the computation, the high values of the hyperpolarizabilities of the title molecule are probably attributed to the charge transfer existing between the phenyl rings within the molecular skeleton. This is evidence for the nonlinear optical (NLO) property of the molecule.

\subsection{Molecular electrostatic potentials (MEP)}

The MEP and electrostatic potential are useful quantities to illustrate the charge distributions of molecules and used to visualize variably charged regions of a molecule. Therefore, the charge distributions can give information about how the molecules interact with another molecule. MEP is widely used as a reactivity map displaying the most probable regions for the electrophilic attack of charged point-like reagents on organic molecule $[72,73]$. The molecular electrostatic potential $\mathrm{V}(r)$ at a given point $r(x, y, z)$ in the vicinity of a molecule, is defined in terms of the interaction energy between the electrical charge generated from the molecule electrons and nuclei and positive test charge (a proton) located at $r$ [74]. Unlike many of the other quantities used at present and earlier as indices of reactivity, $V(r)$ is a real physical property that can be determined experimentally by diffraction or computational methods. For the systems studied, the MEP values were calculated as previously described, using the following equation [75]:

$$
\mathrm{V}(r)=\sum Z_{A} /\left(R_{A}-r\right)-\int \rho\left(r^{\prime}\right) /\left(r^{\prime}-r\right) d r^{\prime}
$$

where the summation runs over all the nuclei $\mathrm{A}$ in the molecule and polarization and reorganization effects are neglected. $Z_{A}$ is the charge of the nucleus $A$, located at $R_{A}$ and $\rho\left(r^{\prime}\right)$ is the electron density function of the molecule.

It provides a visual method to understand the relative polarity of the molecule, as shown in Figure 5 . The different values of the electrostatic potential are represented by different colors; red represented the regions of the most negative electrostatic potential, white represents the regions of the most positive electrostatic potential and blue represents the region of zero potential. The potential increases in the order red < green < blue < pink < white. It can be seen that the negative regions are mainly over the $\mathrm{O}_{7}$ and $\mathrm{O}_{8}$ atoms. The negative (red color) and positive (white) regions of the MEP are related to the electrophilic and nucleophilic reactivity. The predominance of the light green region MEP surface corresponds to a potential half way between two extremes red and white color. The negative electrostatic potential corresponds to an attraction of the proton by the aggregate electron density in the molecule (shades of red), while the positive electrostatic potential corresponds to the repulsion of the protons by the atomic nuclei (shades of white). According to the calculated results, the MEP map shows that the negative potential sites are on oxygen atoms and the positive potential sites are also around the hydrogen atoms. These sites give information about the region from where the compound can have non-covalent interactions.

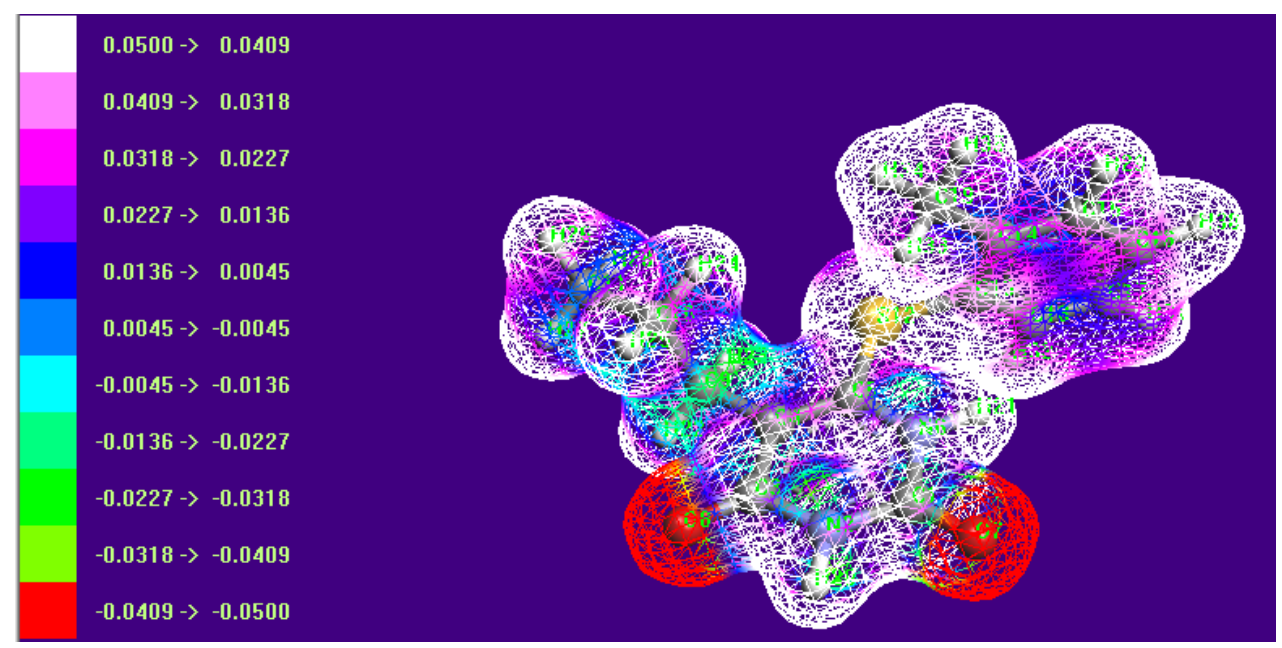

Fig. 5. The total electron density surface mapped with electrostatic potential of the title compound 


\subsection{Total, sum of alpha plus beta electrons (DOS)}

The consideration of only HOMO and LUMO may not yield a realistic description of the frontier orbitals because, in the boundary region, neighboring orbitals may show quasi-degenerate energy levels. For this reason, the total (TDOS), sum of $\alpha$ and $\beta$ electron density of states, in terms of Mulliken population analysis, are calculated and created by convoluting the molecular orbital information with the Gussian curves of unit height and full width at half maximum (FWHM) of 0.3 $\mathrm{eV}$ by using the Gauss Sum 2.2 program [76,77]. The TDOS and $\alpha \beta$ DOS of the title molecule are plotted in Figures 6 and 7.

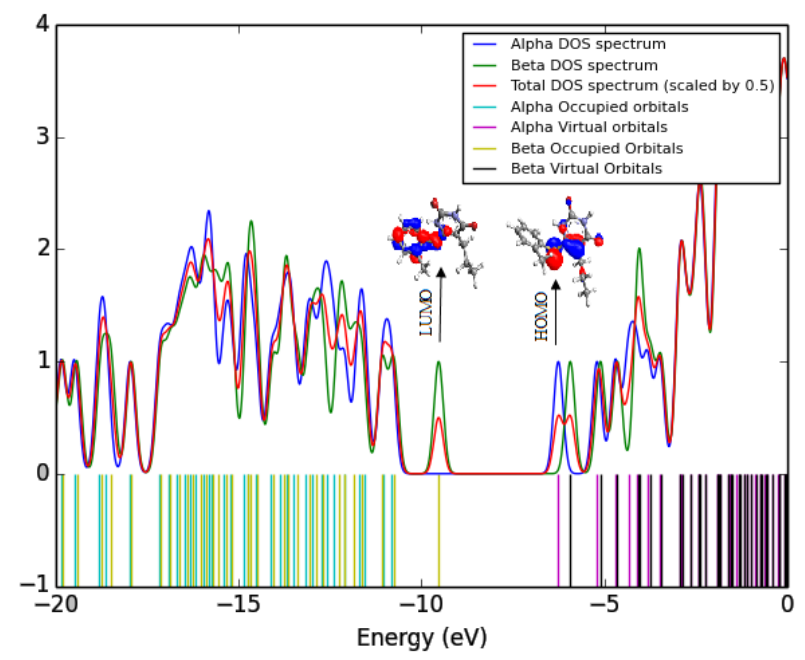

Fig. 6. The sum of alpha plus beta electrons DOS diagram of the title compound

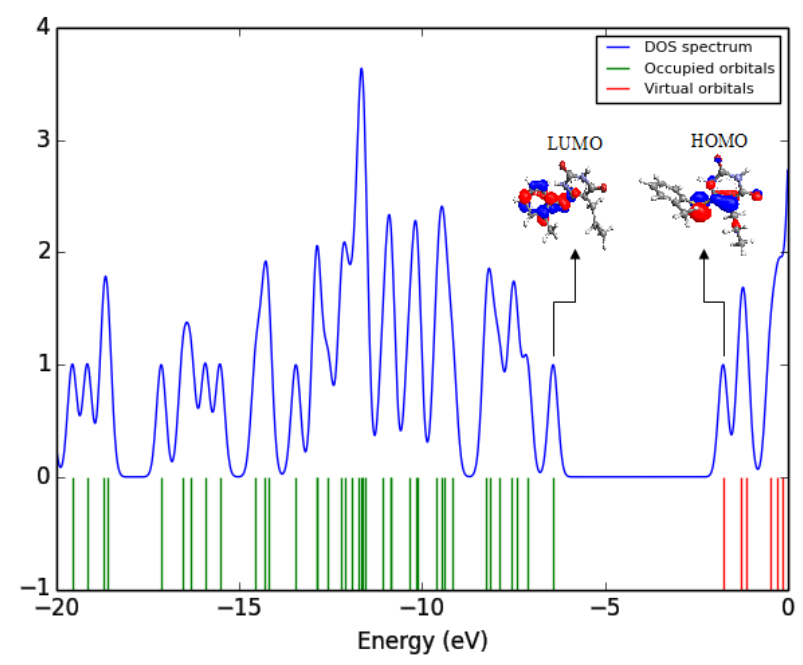

Fig. 7. Calculated total electronic density of states (TDOS) for the title compound
They provide a pictorial representation of molecule orbital (MO) compositions and their contributions to the chemical bonding. The most important application of the DOS plots is to demonstrate the MO compositions and their contributions to the chemical bonding through the positive and negative charges provided by $\alpha \beta$ DOS and TDOS diagrams. The $\alpha \beta$ DOS shows the bonding and sum of positive and negative electrons with the nature of the interaction of the two orbitals, atoms or groups. In this case, the title molecule consists of $73 \alpha$-electrons and $73 \beta$-electrons; in total 146 electrons are occupied in density of states. The way we designate a pictorial representation for cations and anions is essentially similar to that for neutral atoms in their ground state. A positive value of the $\alpha \beta$ DOS indicates a bonding interaction, negative values mean that there is an anti-bonding interaction and zero values indicate non-bonding interactions [78].

\subsection{Thermodynamic properties}

On the basis of vibrational analysis, the statically thermodynamic functions: heat capacity $\left(C_{p, m}^{0}\right)$, entropy $\left(S_{m}^{0}\right)$ and enthalpy changes $\left(H_{m}^{0}\right)$ were computed using the B3LYP/6-311++G(d,p) basis set by using Perl script THERMO.PL and are listed in Table 7. From the results in Table 7, it can be observed that the values of all the thermodynamic functions are increasing with the temperature ranging from 100 to $1000 \mathrm{~K}$ due to the fact that the molecular vibration intensities increase with temperature. The correlation equation between heat capacities, entropies, enthalpy changes and temperatures were fitted by quadratic formulas and the corresponding fitting factors $\left(\mathrm{R}^{2}\right)$; these thermodynamic properties are 0.9999, 0.9995 and 0.9994, respectively. The correlations plot of those shown in Figure 8. The thermodynamic correlation fitting equations are as follows:

$$
\begin{gathered}
\left(C_{p, m}^{0}\right)=273.02+1.1957 \mathrm{~T}-2.8756 \times 10^{-4} \mathrm{~T}^{2} \\
\left(\mathrm{R}^{2}=0.9999\right) \\
\left(S_{m}^{0}\right)=28.374+1.05038 \mathrm{~T}-4.2828 \times 10^{-4} \mathrm{~T}^{2} \\
\left(\mathrm{R}^{2}=0.9995\right) \\
\left(H_{m}^{0}\right)=-12.228+0.1365 \mathrm{~T}-2.9108 \times 10^{-4} \mathrm{~T}^{2} \\
\left(\mathrm{R}^{2}=0.9994\right)
\end{gathered}
$$


T a ble 7

Thermodynamic properties for the title compound (B3LYP/6-311++G(d,p)

\begin{tabular}{cccc}
\hline \hline $\mathrm{T}(\mathrm{K})$ & $C_{p, m}^{0}(\mathrm{~J} / \mathrm{mol} \mathrm{K})$ & $S_{m}^{0}(\mathrm{~J} / \mathrm{mol} \mathrm{K})$ & $H_{m}^{0}(\mathrm{~kJ} / \mathrm{mol})$ \\
\hline 100 & 385.41 & 134.66 & 8.68 \\
200 & 503.88 & 216.7 & 26.24 \\
298 & 605.83 & 299.66 & 51.57 \\
300 & 607.69 & 301.22 & 52.12 \\
400 & 705.57 & 381.65 & 86.34 \\
500 & 798.4 & 450.83 & 128.07 \\
600 & 885.8 & 507.66 & 176.09 \\
700 & 967.66 & 554.12 & 229.26 \\
800 & 1044.24 & 592.49 & 286.65 \\
900 & 1115.93 & 624.59 & 347.55 \\
1000 & 1183.18 & 651.71 & 411.4 \\
\hline \hline
\end{tabular}

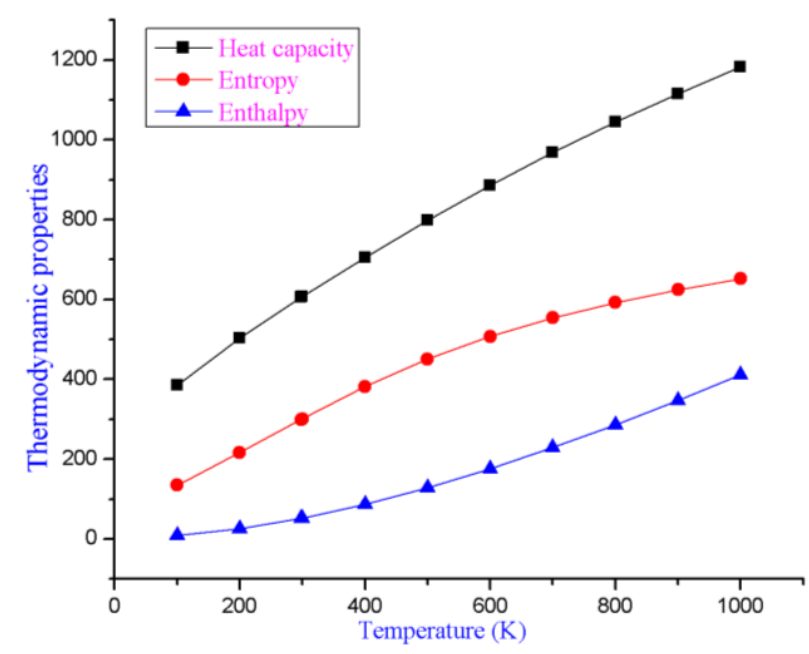

Fig. 8. Correlation plot of the thermodynamic properties at different temperature of the title compound
The thermodynamic data provide very helpful information for further investigation of the title molecule. They can be used to compute the thermodynamic energy according to the relationship between thermodynamic functions and to evaluate the directions of chemical behavior according to the second law of thermodynamics in the thermochemical field [79].

\subsection{Molecular docking studies}

Molecular docking has recently been used as a convenient tool to obtain insights into the molecular mechanism of protein-ligand interactions. Molecular docking studies were carried out to evaluate the binding affinity of the ligand with the active site of c-south African HIV-1 protease (C-SA HIV PR) [80]. The title molecule was chosen to be docked into the active site of different receptors 1HXW, 1D4H, 1D4J and 1EBW anti-Human immune deficiency virus activity against HIV-1 Protease (HIV-PR) obtained from Protein Data Bank (PDB). The docking calculations were carried out using the AutoDock 4.2 package software [81]. The Lamarckian genetic algorithm [82] was applied to search for protein-ligand interaction with and for visualization performed using PyMOL [83]. The ligand was docked into the functional sites of the respective proteins individually and the docking energy was examined to achieve a minimum value. AutoDock results indicated the binding position and bound conformation of the peptide, together with a rough estimate of its interaction. The docked conformation which had the lowest binding energy was chosen to investigate the mode of binding.

Table 8

Binding energy, bonded residues and donor-acceptor bond lengths of the title compound against various protein targets

\begin{tabular}{|c|c|c|c|c|c|c|}
\hline Ligand & $\begin{array}{l}\text { PDB code } \\
\text { for targets }\end{array}$ & Bonded residues & $\begin{array}{c}\text { Estimated } \\
\text { inhibition } \\
\text { Constant } \\
(\mu \mathrm{m})\end{array}$ & $\begin{array}{c}\text { Binding } \\
\text { energy } \\
(\mathrm{Kcal} / \mathrm{mol})\end{array}$ & $\begin{array}{c}\text { Reference } \\
\text { RMSD } \\
(\AA)\end{array}$ & $\begin{array}{l}\text { Donor- acceptor bond } \\
\text { lengths }(\AA)\end{array}$ \\
\hline \multirow{3}{*}{$\begin{array}{l}\text { Title } \\
\text { compound }\end{array}$} & \multirow{3}{*}{ 1HXW } & ASP $29(\mathrm{NH} . . . \mathrm{O})$ & \multirow{3}{*}{908.70} & \multirow{3}{*}{-4.15} & \multirow{3}{*}{24.75} & 2.5 \\
\hline & & ASP $29($ O...O $)$ & & & & 2.9 \\
\hline & & ASP $29(\mathrm{O} \ldots \mathrm{HN})$ & & & & 2.2 \\
\hline \multirow{3}{*}{$\begin{array}{c}\text { Title } \\
\text { compound }\end{array}$} & \multirow{3}{*}{$1 \mathrm{D} 4 \mathrm{H}$} & ILE $50(\mathrm{O} \ldots \mathrm{HN})$ & \multirow{3}{*}{24.35} & \multirow{3}{*}{-6.29} & \multirow{3}{*}{24.40} & 2.2 \\
\hline & & ILE $50(\mathrm{O} \ldots \mathrm{HN})$ & & & & 1.9 \\
\hline & & ASP $125(\mathrm{NH} \ldots \mathrm{O})$ & & & & 1.9 \\
\hline \multirow{6}{*}{$\begin{array}{l}\text { Title } \\
\text { compound }\end{array}$} & \multirow{6}{*}{ 1D4J } & ASP $130(\mathrm{O} \ldots \mathrm{O})$ & \multirow{6}{*}{9.64} & \multirow{6}{*}{-6.84} & \multirow{6}{*}{23.97} & 2.7 \\
\hline & & ASP $130(\mathrm{O} \ldots \mathrm{N})$ & & & & 2.9 \\
\hline & & ASP $129(\mathrm{NH} . . \mathrm{O})$ & & & & 2.5 \\
\hline & & ASP $129(\mathrm{O} \ldots \mathrm{O})$ & & & & 2.8 \\
\hline & & ARG 8 (O...NH) & & & & 2.6 \\
\hline & & GLY 148 (NH...O) & & & & 2.2 \\
\hline \multirow{2}{*}{$\begin{array}{c}\text { Title } \\
\text { compound }\end{array}$} & \multirow{2}{*}{ 1EBW } & ASP $25(\mathrm{NH} . . . \mathrm{O})$ & \multirow{2}{*}{133.72} & \multirow{2}{*}{-5.28} & \multirow{2}{*}{26.22} & 2.1 \\
\hline & & ILE $50(\mathrm{O} \ldots \mathrm{HN})$ & & & & 2.0 \\
\hline
\end{tabular}




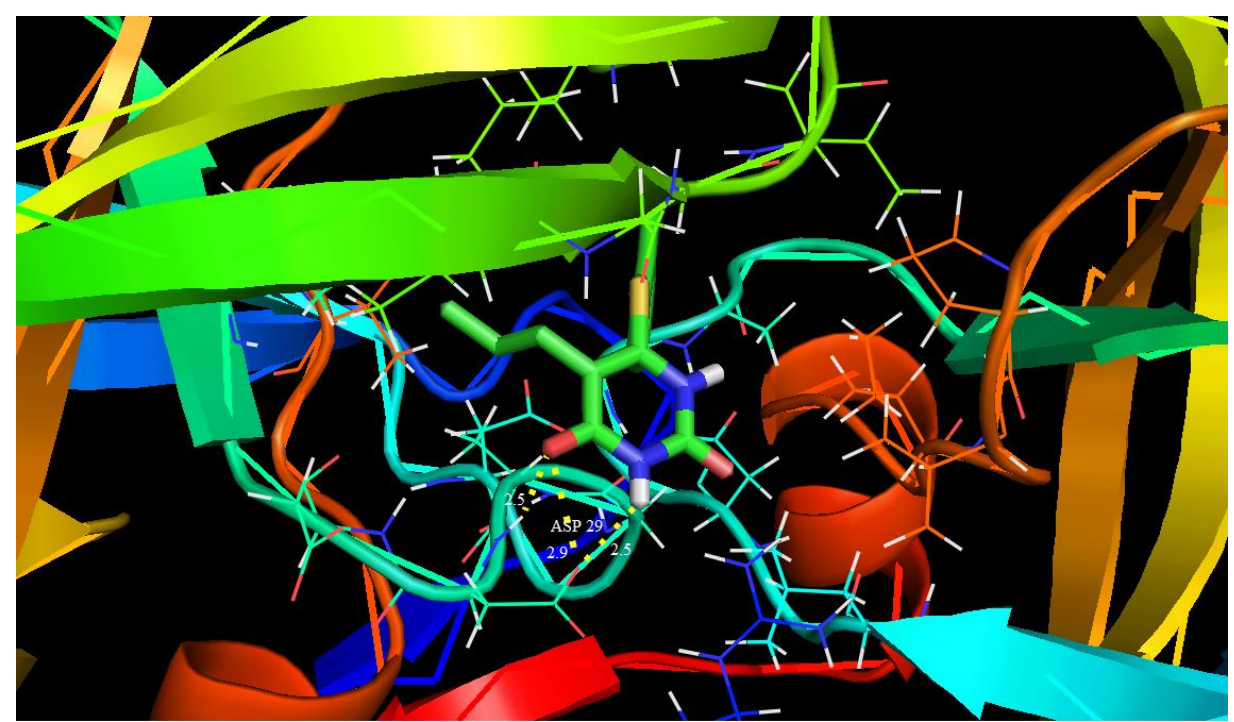

Fig. 9. Docking and hydrogen bond interactions of the title compound with chain A,B of 1HXW protein structure

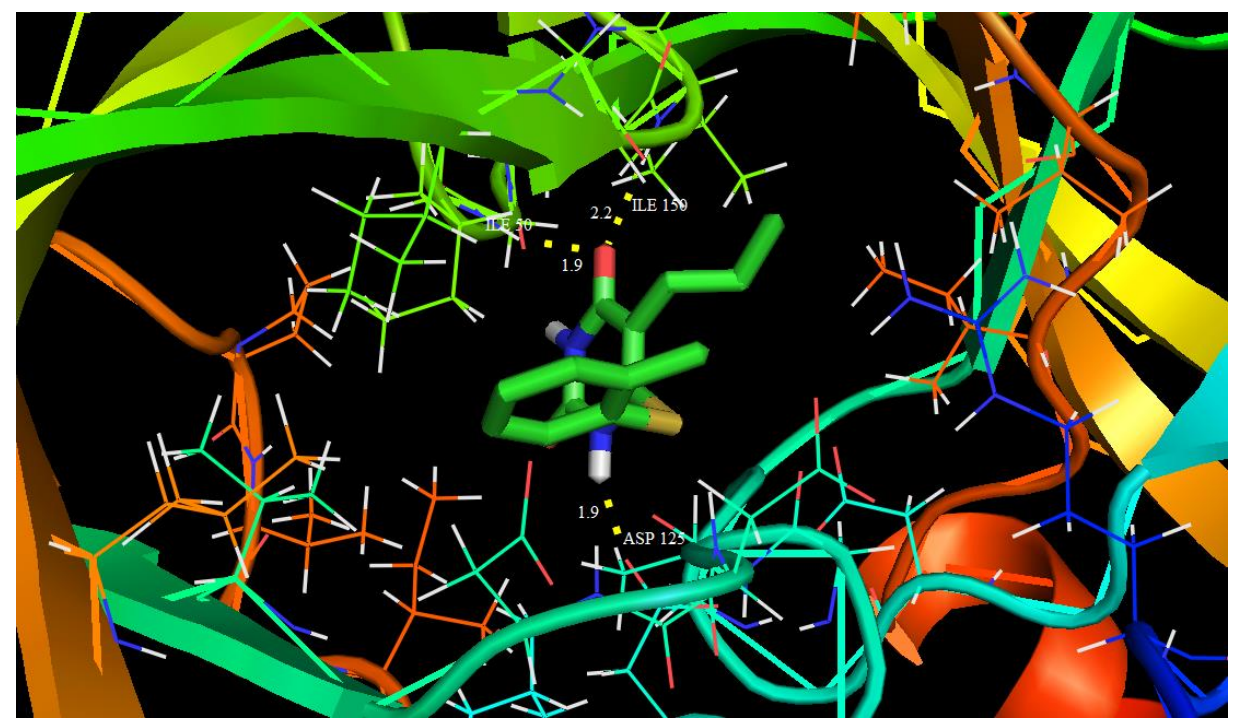

Fig. 10. Docking and hydrogen bond interactions of the title compound with chain A,B of 1D4H protein structure

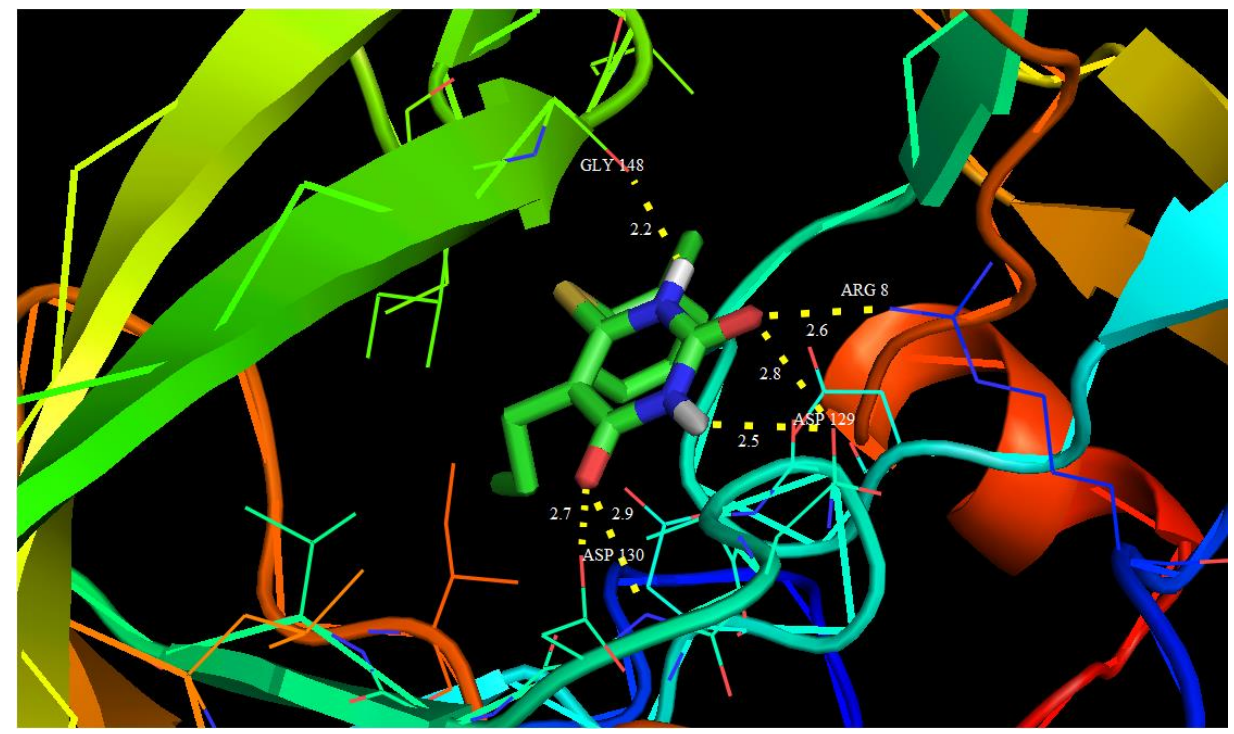

Fig. 11. Docking and hydrogen bond interactions of the title compound with chain A,B of 1D4J protein structure 


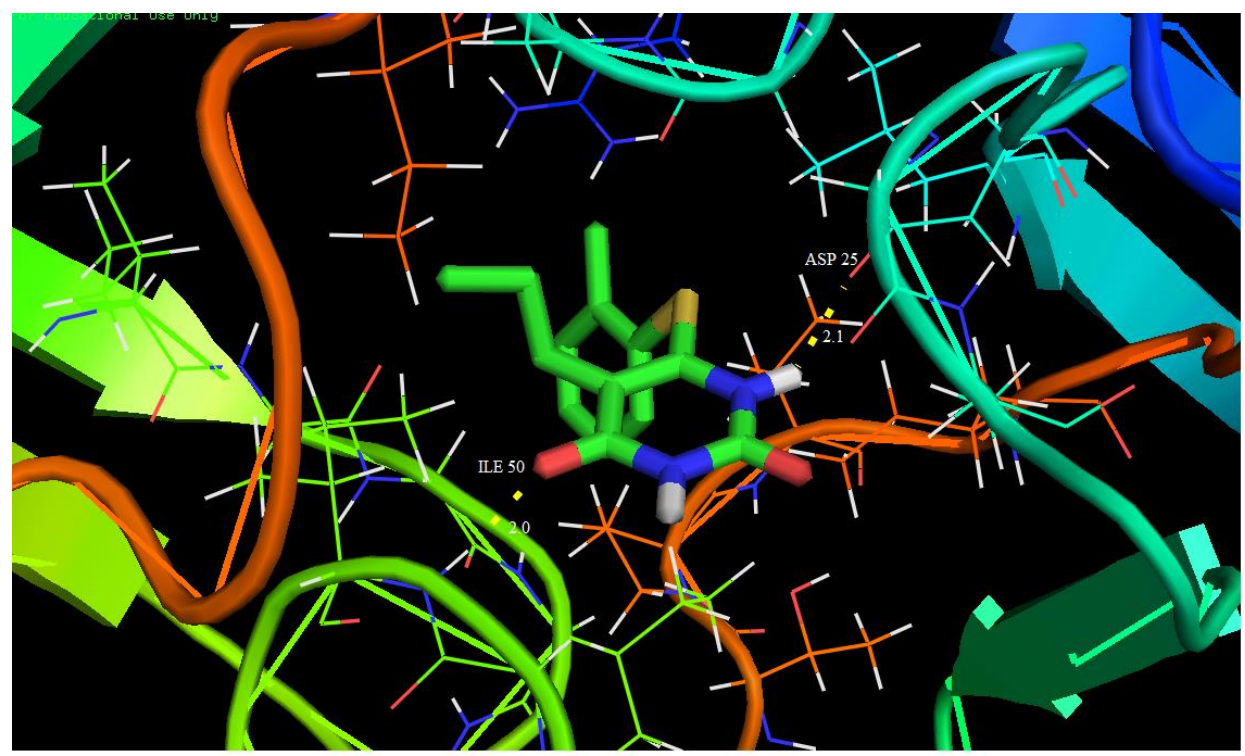

Fig. 12. Docking and hydrogen bond interactions of the title compound with chain $1 \mathrm{EBW}$ protein structure

The molecular docking binding energies $(\mathrm{kcal} / \mathrm{mol})$ and inhibition constants $(\mu \mathrm{m})$ were also obtained and listed in Table 8. Among them, 1D4J exhibited the lowest free energy at $-6.84 \mathrm{kcal} / \mathrm{mol}$ and most docked inhibitors interacted with the ligand within the 1D4J binding site. They exhibited up to six donor- acceptor interactions involving two ASP 130, two ASP 129, one ARG 8 and one GLY 148 with an RMSD of $23.97 \AA$. The docking simulation shows the binding mode of the title compound into 1D4J. The ligand interactions with different receptors are shown in Figures 9-12.

\section{CONCLUSIONS}

In the present study, the title compound, namely 6-[(2-methylphenyl)sulfanyl]-5-propylpyrimidine-2,4(1H,3H)-dione, is theoretically optimized using the B3LYP method with the 6$311++\mathrm{G}(\mathrm{d}, \mathrm{p})$ basic set and the optimized geometry is compared with the experimental XRD data and well discussed. The vibrational FT-IR and FTRaman spectra of compounds were recorded; a detailed description of vibrational modes was assigned with the aid of normal coordinate analysis. The contributions to each of the observed frequencies showed the reliability and accuracy of the spectral analysis. The NBO analysis revealed that there is an efficient intramolecular charge transfer (ICT) within the molecule. The MEP map showed that the negative potential sites are on oxygen and nitrogen atoms, and the positive potential sites are also the NH hydrogen atoms. The TDOS and $\alpha \beta$ DOS, were also calculated. The calculated differ- ence in the HOMO and LUMO energies supported the charge transfer occurring within the molecule. The Fukui function and the first order hyperpolarizability were determined. The thermodynamic functions of the molecule at different temperatures have been calculated. The heat capacities, entropies and enthalpies increased with increasing temperature owing to the intensities of the molecular vibrations. Moreover, the molecular docking simulations have been performed to predict the biological inhibition activity against HIV-1 protease. The results showed that the title molecule has potential activity against HIV-1 protease.

Acknowledgment. The authors extend their appreciation to the deanship of scientific research and the research center, college of pharmacy, King Saud University for funding this research.

\section{REFERENCES}

[1] R. Ragno, A. Mai, S. Sbardella, M. Artico, S. Massa, C. Musiu, M. Mura, T. Marceddu, A. Cadeddu, P. La Colla, Computer-aided design, synthesis, and anti-HIV-1 activity in vitro of 2-alkylamino-6-[1-(2,6-difluorophenyl)alkyl]3,4-dihydro-5-alkylpyrimidin-4(3H)-ones as novel potent non-nucleoside reverse transcriptase inhibitors also active against the Y181C variant, J. Med. Chem. 47, 928934 (2004). DOI: 10.1021/jm0309856

[2] X. Lu, Y. Chen, Y. Guo, Z. Liu, Y. Shi, Y. Xu, X. Wang, Z. Zhang, J. Liu, The design and synthesis of N1-alkylated-5-aminoaryalkylsubstituted-6-methyluracils as potential non-nucleoside HIV-1 RT inhibitors, Bioorg. Med. Chem. 15, 7399-7407 (2007). DOI: 10.1016/j.bmc.2007.07.058

[3] A. A. El-Emam, M. A. Massoud, E. R. El-Bendary, M. A. El-Sayed, Synthesis of certain 6-substituted uracils 
and related derivatives as potential antiviral agents, Bull. Kor. Chem. Soc. 25, 991-996 (2004).

DOI: $10.5012 / \mathrm{bkcs} .2004 .25 .7 .991$

[4] N. R. El-Brollosy, O. A. Al-Deeb, A. A. El-Emam, E. B. Pedersen, P. La Colla, G. Collu, G. Sanna, L. Roberta, Synthesis of novel uracil non-nucleoside derivatives as potential reverse transcriptase inhibitors of HIV-1, Arch. Pharm. 342, 663-670 (2009).

DOI: $10.1002 / \operatorname{ardp} .200900139$

[5] M. Artico, S. Massa, A. Mai, M.E. Marongiu, G. Piras, E. Tramontino, P. La Colla, 3,4-Dihydro-2-alkyloxy-6benzyl-4-oxoypyrimidines (DABOs): a new class of specific inhibitors of human immunodeficiency virus type 1, Antiviral Chem. Chemother. 4, 361-368 (1993). DOI: $10.1177 / 095632029300400608$

[6] R. Kumar, W. Semaine, M. Johar, D. L. J. Tyrrell, B. Agrawal, Effect of various pyrimidines possessing the 1[(2-hydroxy-1-(hydroxymethyl)ethoxy)methyl] moiety, able to mimic natural $2^{6}$-deoxyribose, on wild-type and mutant hepatitis B virus replication, J. Med. Chem. 49, 3693-3700 (2006). DOI: 10.1021/jm010410d

[7] M. N. Brunelle, J. Lucifora, J. Neyts, S. Villet, A. Holy, C. Trepo, F. Zoulim, In vitro activity of 2,4-diamino-6[2-(phosphonomethoxy)ethoxy]-pyrimidine against multidrug-resistant hepatitis B virus mutants, Antimicrob. Agents Chemother. 51, 2240-2243 (2007).

DOI: 10.1128/AAC.00138-06

[8] Y. Ding, J. L. Girardet, K. L. Smith, G. Larson, B. Prigaro, J. Z. Wu, N. Yao, Parallel synthesis of 5-cyano6-aryl-2-thiouracil derivatives as inhibitors for hepatitis C viral NS5B RNA-dependent RNA polymerase, Bioorg. Chem. 34, 26-38 (2006).

DOI: 10.1016/j.bioorg.2005.10.001

[9] K. K. Gauni, H. Kohlhage, Reassessment of the rationale for the combinations of sulphonamides with diaminopyrimidines, Chemotherapy 14, 158-169 (1969). DOI: $10.1159 / 000220625$

[10] P. Russ, P. Schelling, L. Scapozza, G. Folkers, E. De Clercq, V. E. Marquez, Synthesis and biological evaluation of 5-substituted derivatives of the potent antiherpes agent (north)-methanocarbathymine, J. Med. Chem. 46, 5045-5054 (2003). DOI: 10.1021/jm00128a029

[11] R. S. Klein, M. Lenzi, T. H. Lim, K. A. Hotchkiss, P. Wilson, E. L. Schwartz, Novel 6-substituted uracil analogs as inhibitors of the angiogenic actions of thymidine phosphorylase, Biochem. Pharmacol. 62, 1257-1263 (2001). DOI: 10.1016/S0006-2952(01)00783-3

[12] O. N. Al-Safarjalani, X. Zhou, R. H. Rais, J. Shi, R. F. Schinazi, F. N. M. Naguib, M. H. El Kouni, 5(Phenylthio)acyclouridine: a powerful enhancer of oral uridine bioavailability: relevance to chemotherapy with 5-fluorouracil and other uridine rescue regimens, Cancer Chemother. Pharmacol. 55, 541-551 (2005). DOI: $10.1007 / \mathrm{s} 00280-004-0967-\mathrm{y}$

[13] K. Ghoshal, S. T. Jacob, An alternative molecular mechanism of action of 5-fluorouracil, a potent anticancer drug, Biochem. Pharmacol. 53, 1569-1575 (1997). DOI: 10.1016/S0006-2952(97)00040-3

[14] N. Sirisoma, S. Kasibhatla, B. Nguyen, A. Pervin, Y. Wang, G. Claassen, B. Tseng, J. Drewe, S. X. Cai, Discovery of substituted 4-anilino-2-(2-pyridyl)pyrimidines as a new series of apoptosis inducers using a cell- and caspase-based high throughput screening assay. Part 1: Structure-activity relationships of the 4-anilino group, Bioorg. Med. Chem. 14, 7761-7773 (2006).

DOI: $10.1016 / j . b m c .2006 .08 .002$

[15] H. H. Locher, H. Schlunegger, P. G. Hartman, P. Anghern, R. L. Then, Antibacterial activities of epiroprim, a new dihydrofolate reductase inhibitor, alone and in combination with dapsone, Antimicrob. Agents Chemother. 40, 1376-1381 (1996).

[16] C. A. Sincak, A novel diaminopyrimidine for the treatment of resistant Gram-positive infections, Ann. Pharmacother. 43, 1107-1114 (2009). DOI: 10.1345/aph.1L167

[17] E. S. Al-Abdullah, A. A. Al-Turkistani, O. A. Al-Deeb, N. R. El-Brollosy, E. E. Habib, A. A. El-Emam, Pyrimidine-5-carbonitriles, II: Synthesis and antimicrobial activity of novel 6-alkyl-2,4-disubstituted pyrimidine-5carbonitriles, Drug Res. 64, 31-39 (2014). DOI: $10.1055 / \mathrm{s}-0033-1351315$

[18] E. S. Al-Abdullah, A. R. Al-Obaid, O. A. Al-Deeb, E. E. Habib, A. A. El-Emam, Synthesis of novel 6-phenyl-2,4disubstituted pyrimidine-5-carbonitriles as potential antimicrobial agents, Eur. J. Med. Chem. 46, 4642-4647 (2011). DOI: 10.1016/j.ejmech.2011.08.003

[19] W. Brumfitt, J. M. Hamilton-Miller, Reassessment of the rationale for the combinations of sulphonamides with diaminopyrimidines, J. Chemother. 5, 465-469 (1993). DOI: 10.1080/1120009X.1993.11741097

[20] D. Tassel, M. A. Madoff, Treatment of candida sepsis and cryptococcus meningitis with 5-fluorocytosine. A new antifungal agent, J. Am. Med. Assoc. 206, 830-832 (1968). DOI: 10.1001/archinte.1975.00330020035003

[21] A. Mai, D. Rotili, S. Massa, G. Brosch, G. Simonetti, C. Passariello, A. Palamara, Discovery of uracil-based histone deacetylase inhibitors able to reduce acquired antifungal resistance and trailing growth in candida albicans, Bioorg. Med. Chem. Lett. 17, 1221-1225 (2007). DOI: $10.1016 /$ j.bmcl.2006.12.028

[22] A. F. Cowman, M. J. Morry, B. A. Biggs, G. A. Cross, S. J. Foote, Amino acid changes linked to pyrimethamine resistance in the dihydrofolate reductase-thymidylate synthase gene of Plasmodium falciparum, Proc. Natl. Acad. Sci. USA 85, 9109-9113 (1988).

[23] A. Sardarian, K. T. Douglas, M. Read, P. F. G. Sims, J. E. Hyde, P. Chitnumsub, R. Sirawaraporn, W. Sirawaraporn, Pyrimethamine analogs as strong inhibitors of double and quadruple mutants of dihydrofolate reductase in human malaria parasites, Org. Biomol. Chem. 1, 960964 (2003). DOI: 10.1039/B211636G

[24] C. Sirichaiwat, C. Intaraudom, S. Kamchonwongpaisan, J. Vanichtanankul, Y. Thebtaranonth, Y. Yuthavong, Target guided synthesis of 5-benzyl-2,4-diamonopyrimidines: Their antimalarial activities and binding affinities to wild type and mutant dihydrofolate reductases from Plasmodium falciparum, J. Med. Chem. 47, 345354 (2004). DOI: 10.1021/jm0303352

[25] B. K. Singh, M. Mishra, N. Saxena, G. P. Yadav, P. R. Maulik, M. K. Sahoo, R. L. Gaur, P. K. Murthy, R. P. Tripathi, Synthesis of 2-sulfanyl-6-methyl-1,4-dihydropyrimidines as a new class of antifilarial agents, Eur. $J$. Med. Chem. 43, 2717-2723 (2008). DOI: $10.1016 /$ j.ejmech.2008.01.038 
[26] R. Pontikis, R. Benhida, A. H. Aubertin, D. S. Grieson, C. Monneret, Synthesis and anti-HIV activity of novel $N-1$ side chain-modified analogs of 1-[(2-hydroxyethoxy)methyl]-6-(phenylthio)thymine (HEPT), J. Med. Chem. 40, 1845-1854 (1997). DOI: 10.1021/jm960765a

[27] A. L. Hopkins, J. Ren, R. M. Esnouf, B. E. Willcox, E. Y. Jones, C. Ross, T. Miyasaka, R. T. Walker, H. Tanaka, D. K. Stammers, D. I. Stuart, Complexes of HIV-1 reverse transcriptase with Inhibitors of the HEPT Series reveal conformational changes relevant to the design of potent non-nucleoside inhibitors, J. Med. Chem. 39, 1589-1600 (1996). DOI: 10.1021/jm960056x

[28] H. Tanaka, H. Takashima, M. Ubasawa, K. Sekiya, I. Nitta, M. Baba, S. Shigeta, R. T. Walker, E. De Clercq, T. Miyasaka, Synthesis and antiviral activity of deoxy analogs of 1-[(2-hydroxyethoxy)-methyl]-6-(phenylthio) thymine (HEPT) as potent and selective anti-HIV-1 agents, J. Med. Chem. 35, 4713-4719 (1992). DOI: $10.1021 / \mathrm{jm} 00103 \mathrm{a} 009$

[29] T. Miyasaka, H. Tanaka, M. Baba, H. Hayakawa, R. T. Walker, J. Balzarini, E. De Clercq, A novel lead for specific anti-HIV-1 agents 1-[(2-hydroxyethoxy)methyl]-6(phenylthio)thymine, J. Med. Chem. 32, 2507-2509 (1989). DOI: 10.1021/jm00132a002

[30] M. I. Attia, A. A. El-Emam, A. A. Al-Turkistani, A. L. Kansoh, N. R. El-Brollosy, Synthesis of novel 2(substituted amino)alkylthiopyrimidin-4(3H)-ones as potential antimicrobial agents, Molecules 19, 279-290 (2014). DOI: 10.3390/molecules 19010279

[31] N. Z. Alzoman, Y. S. Mary, C. Y. Panicker, I. A. AlSwaidan, A. A. El-Emam, O. A. Al-Deeb, A. A. Al-Saadi, C. Van Alsenoy, J. A. War, Spectroscopic investigation (FT-IR and FT-Raman), vibrational assignments, HOMOLUMO, NBO, MEP analysis and molecular docking study of 2-[(4-chlorobenzyl)sulfanyl]-4-(2-methylpropyl)6-(phenylsulfanyl)-pyrimidine-5-carbonitrile, a potential chemotherapeutic agent, Spectrochim. Acta A 139, 413424 (2015). DOI: 10.1016/j.saa.2014.12.043

[32] N. G. Haress, A. A. El-Emam, O. A. Al-Deeb, C. Y. Panicker, A. A. Al-Saadi, C. Van Alsenoy, J. A. War, S. K. Srivastava, Vibrational spectroscopic and molecular docking study of 2-benzylsulfanyl-4-[(4-methylphenyl)sulfanyl]-6-pentylpyrimidine-5-carbonitrile, a potential chemotherapeutic agent, Spectrochimica Acta A 137, 569-580 (2015). DOI: 10.1016/j.saa.2014.08.112

[33] E. S. Al-Abdullah, Y. S. Mary, C. Y. Panicker, N. R. ElBrollosy, A. A. El-Emam, C. Van Alsenoy, A. A. AlSaadi, Theoretical investigations on the molecular structure, vibrational spectra, HOMO-LUMO analyses and NBO study of 1-[(cyclopropylmethoxy)methyl]-5-ethyl-6(4-methylbenzyl)-1,2,3,4-tetrahydropyrimidine-2,4-dione, Spectrochim. Acta A 133, 639-650 (2014). DOI: 10.1016/j.saa.2014.06.042

[34] Y. Sert, A. A. El-Emam, O. A. Al-Deeb, A. A. AlTurkistani, Ç. Çirak, F. Ucun, The biomolecule, 2-[(2methoxyl)sulfanyl]-4-(2-methylpropyl)-6-oxo-1,6-dihydropyrimidine-5-carbonitrile: FT-IR, Laser-Raman spectra and DFT, Spectrochim. Acta A 126, 86-97 (2014). DOI: 10.1016/j.saa.2014.01.140

[35] M. A. Al-Alshaikh, O. A. Al-Deeb, N. Z. Alzoman, A. A. El-Emam, R. Srivastava, A. K. Sachan, O. Prasad, L. Sinha, Spectroscopic and electronic structure calculation of a potential chemotherapeutic agent 5-propyl-6-(p-
tolylsulfanyl)pyrimidine-2,4(1H,3H)-dione using first principles, J. Mol. Struct. 1100, 225-226 (2015).

DOI: 10.1016/j.molstruc.2015.07.042

[36] N. G. Haress, H. A. Ghabbour, A. A. El-Emam, C. S. Chidan Kumar, H.-K. Fun, 6-[(2-Methylphenyl) sulfanyl]-5-propylpyrimidine-2,4(1H,3H)-dione, Acta Cryst. E70, o768-o769 (2014).

DOI: $10.1107 / \mathrm{S} 1600536814013269$

[37] S. S. Gupta, A. Marchno, R. D. Pradhan, C. F. Desai, J. Melikechi, Pump-probe thermal lens near-infrared spectroscopy and Z-scan study of zinc (tris) thiourea sulfate, J. Appl. Phys. 89, 4939-4943 (2001). DOI: $10.1063 / 1.135825$

[38] S. P. Karna, Electronic and nonlinear optical materials: The role of theory and modeling, J. Phys. Chem. A 104, 4671-4673 (2000). DOI: 10.1021/jp001296y

[39] D. R. Kanis, M. A. Ratner, T. J. Marks, Design and construction of molecular assemblies with large secondorder optical nonlinearities. Quantum chemical aspects, Chem. Rev. 94, 195-242 (1994). DOI: $10.1021 / \mathrm{cr} 00025 \mathrm{a} 007$

[40] H. S. Nalwa, S. Miyata, Nonlinear Optical Properties of Organic Molecules and Polymers, CRC Press, Boca Raton, FL, 1996.

[41] M. Govindarajan, M. Karbacak, V. Udayakumar, S. Periandy, FT-IR, FT-Raman and UV spectral investigation: Computed frequency estimation analysis and electronic structure calculations on chlorobenzene using $\mathrm{HF}$ and DFT, Spectrochim. Acta A 88, 37-48 (2012). DOI: $10.1016 /$ j.saa.2011.11.052

[42] Gaussian Inc, Gaussian 03 Program, Guassion Inc., Wallingford, CT, 2004.

[43] H. B. Schlegel, Optimization of equilibrium geometries and transition structures, J. Comput. Chem. 3, 214-218 (1982). DOI: $10.1002 / j$ cc.540030212

[44] M. H. Jamroz, Vibrational Energy Distribution Analysis: VEDA 4 Program, Warsaw, 2004.

[45] A. E. Reed, F. Weinhold, Natural localized molecular orbitals, J. Chem. Phys. 83, 1736-1740 (1985). DOI: $10.1063 / 1.449360$

[46] G. Keresztury, S. Holly, G. Besenyei, J. Varga, A. Y. Wang, J. R. Durig, Vibrational spectra of monothiocarbamates-II. IR and Raman spectra, vibrational assignment, conformational analysis and $a b$ initio calculations of $S$-methyl- $N, N$-dimethylthiocarbamate, Spectrochim. Acta A 49, 2007-2017 (1993). DOI: $10.1016 / \mathrm{S} 0584-8539(09) 91012-1$

[47] G. Keresztury, J. M. Chalmers, P. R. Griffith (Eds.), Raman Spectroscopy: Theory - Handbook of Vibrational Spectroscopy, John Wiley \& Sons, New York, 2002.

[48] Y. Wang, S. Saebø, C. U. Pittman Jr., The structure of aniline by ab initio studies, J. Mol. Struct. THEOCHEM. 281, 91-98 (1993). DOI: $10.1016 / 0166-1280(93) 87064-K$

[49] G. Socrates, Infrared and Raman Characteristic Frequencies, Third ed., John Wiley \& Sons Ltd., Chichester, 2001.

[50] L. J. Bellamy, The Infrared Spectra of Complex Molecule, Third ed., Wiley, New York, 1975. 
[51] M. Pagannone, B. Fornari, G. Mattei, Molecular structure and orientation of chemisorbed aromatic carboxylic acids: Surface enhanced Raman spectrum of benzoic acid adsorbed on silver sol, Spectrochim. Acta 43, 621625 (1987). DOI: 10.1016/0584-8539(87)80143-5

[52] P. S. Patil, S. M. Dharmaprakash, Synthesis, growth and characterization of second-order nonlinear optical crystal: 5-Br-2-thienyl-4'-methoxychalcone, J. Cryst. Growth, 305, 218-221 (2007).

DOI: $10.1016 / j$.jcrysgro.2007.04.038

[53] N. B. Colthup, L. H. Daly, S. E. Wiberley, Introduction to Infrared and Raman Spectroscopy, $3^{\text {rd }}$. ed., Academic Press, Boston, MA, 1990.

[54] N. P. Roeges, A Guide to the Complete Interpretation of Infrared Spectra of Organic Structures, Wiley, New York, 1994

[55] G. Litivinow, Proceedings of the XII International Conference on Raman Spectroscopy, Wurzburg, Germany, 1992.

[56] M. Siva Priya, T. A. Benitta, C. James, Vibrational spectroscopic investigation and normal coordinate analysis of the fibrate hypolipidemic agent 5-(2,5-dimethylphenoxy)-2,2-dimethyl pentanoic acid (Gemfibrozil), J. Mol. Struct. 990, 253-262 (2011).

DOI: 10.1016/j.molstruc.2011.01.054

[57] M. Kandasamy, G. Velraj, Ab initio/DFT electronic structure calculations, spectroscopic studies of 5-bromo2-pyridinecarbonitrile - A comparative study, Solid State Sci. 14, 1071-1079 (2012).

DOI: $10.1016 /$ j.solidstatesciences.2012.05.003

[58] G. Gece, The use of quantum chemical methods in corrosion inhibitor studies, Corros. Sci. 50, 2981-2992 (2008). DOI: 10.1016/j.corsci.2008.08.043

[59] D. F. V. Lewis, C. Loannides, D. V. Parke, Interaction of a series of nitriles with the analysis of structureactivity relationships, Xenobiotica 24, 401-408 (1994). DOI: $10.3109 / 00498259409043243$

[60] P. Kolandaivel, G. Praveen, P. Selvarengan, Study of atomic and condensed atomic indices for reactive sites of molecules, J. Chem. Sci. 117, 591-598 (2005). DOI: $10.1007 / \mathrm{BF} 02708366$

[61] S. Muthu, E. Isac Paulraj, Spectroscopic and molecular structure (monomeric and dimeric structure) investigation of 2-[(2-hydroxyphenyl)carbonyloxy]benzoic acid by DFT method: A combined experimental and theoretical study, J. Mol. Struct. 1038, 145-162 (2013). DOI: $10.1016 /$ j.molstruc.2013.01.043

[62] W. Yang, W. J. Mortier, The use of global and local molecular parameters for the analysis of the gas-phase basicity of amines, J. Am. Chem. Soc. 108, 5708-5711 (1986). DOI: $10.1021 / \mathrm{ja} 00279 \mathrm{a} 008$

[63] Y. -X. Sun, Q. -L. Hao, W. -X. Wei, Z. -X. Yu, L. -D. Lu, X. Wang, Y. -S. Wang, Experimental and density functional studies on 4-(3,4-dihydroxybenzylideneamino)antipyrine, and 4-(2,3,4-trihydroxybenzylideneamino)antipyrine, J. Mol. Struct. THEOCHEM 904, 7482 (2009). DOI: 10.1016/j.theochem.2009.02.036

[64] H. W. Thomson, P. Torkington, The vibrational spectra of esters and ketones, J. Chem. Soc. 171, 640-645 (1945). DOI: 10.1039/JR9450000640
[65] M. Szafran, A. Komasa, E. B. Adamska, Crystal and molecular structure of 4-carboxypiperidinium chloride (4-piperidinecarboxylic acid hydrochloride), J. Mol. Struct. THEOCHEM 827, 101-107 (2007).

DOI: 10.1016/j.molstruc.2006.05.012

[66] J. Liu, Z. Chen, S. Yuan, Study on the prediction of visible absorption maxima of azobenzene compounds, $J$. Zhejiang Univ. Sci. B. 6, 584-589 (2005). DOI: $10.1631 /$ jzus.2005.B0584

[67] M. Nakano, H. Fujita, M. Takahata, K. Yamaguchi, Theoretical study on second hyperpolarizabilities of phenylacetylene dendrimer: Toward an understanding of structure-property relation in NLO responses of fractal antenna dendrimers, J. Am. Chem. Soc. 124, 96489655 (2002). DOI: 10.1021/ja0115969

[68] D. Sajan, I. Joe, V. S. Jayakumar, J. Zaleski, Structural and electronic contributions to hyperpolarizability in methyl $p$-hydroxy benzoate, J. Mol. Struct. 785, 43-53 (2006). DOI: 10.1016/j.molstruc.2005.09.041

[69] K. S. Thanthiriwatte, K. M. N. de Silva, Non-linear optical properties of novel fluorenyl derivatives $-a b$ initio quantum chemical calculations, J. Mol. Struct. THEOCHEM 617, 169-175 (2002). DOI: $10.1016 / \mathrm{S} 0166-1280(02) 00419$

[70] S. G. Sagdinc, A. Esme, Theoretical and vibrational studies of 4,5-diphenyl-2-2 oxazole propionic acid (oxaprozin), Spectrochim. Acta A 75, 1370-1376 (2010). DOI: $10.1016 /$ j.saa.2010.01.004

[71] S. Muthu, G. Ramachandran, Spectroscopic studies (FTIR, FT-Raman and UV-Visible), normal coordinate analysis, NBO analysis, first order hyper polarizability, HOMO and LUMO analysis of (1R)- $N$-(prop-2-yn-1yl)-2,3-dihydro- $1 H$-inden-1-amine molecule by $a b$ initio $\mathrm{HF}$ and density functional methods, Spectrochim. Acta A 121, 394-403 (2014). DOI: 10.1016/j.saa.2013.10.093

[72] S. Muthu, T. Rajamani, M. Karabacak, A. M. Asiri, Vibrational and UV spectra, first order hyperpolarizability, NBO and HOMO-LUMO analysis of 4-chloro- $\mathrm{N}$-(2methyl-2,3-dihydroindol-1-yl)-3-sulfamoyl-benzamide, Spectrochim. Acta A 122, 1-14 (2014). DOI: 10.1016/j.saa.2013.10.115

[73] P. Politzer, D. G. Truhlar (Eds.), Chemical Application of Atomic and Molecular Electrostatic Potentials, Plenum, New York, 1981

[74] E. E. Porchelvi, S. Muthu, The spectroscopic (FT-IR, FT-Raman and NMR), NCA, Fukui function analysis first order hyperpolarizability, TGA of 6-chloro3,4dihydro-2H-1,2,4-benzothiazine-7-sulphonamide1,1dioxide by $a b$ initio HF and Density Functional method, Spectrochim. Acta A 123, 230-240 (2014). DOI: $10.1016 /$ j.saa.2013.12.037

[75] P. Politzer, J. Murray, The fundamental nature and role of the electrostatic potential in atoms and molecules, Theor. Chem. Acc. 108, 134-142 (2002). DOI: $10.1007 / \mathrm{s} 00214-002-0363-9$

[76] N. M. O’Boyle, A. L. Tenderholt, K. M. Langer, cclib: A library for package-independent computational chemistry algorithms, J. Comput. Chem. 29, 839-845 (2008). DOI: $10.1002 /$ jcc. 20823

[77] S. Muthu, G. Ramachandran, E. I. Paulraj, T. Swaminathan, Quantum mechanical study of the structure and 
spectroscopic (FTIR, FT-Raman), first-order hyperpolarizability and NBO analysis of 1,2-benzoxazol-3ylmenthane sulfonamide, Spectrochim. Acta A 128, 603613 (2014). DOI: 10.1016/j.saa.2014.02.183

[78] M. Chen, U. V. Waghmare, C. M. Friend, F. Kaxiras, A density functional study of clean and hydrogen-covered $\alpha$-MoO3 (010): Electronic structure and surface relaxation, J. Chem. Phys. 109, 6680-6854 (1998). DOI: $10.1063 / 1.477252$

[79] S. Muthu, E. I. Palulraj, Spectroscopic and molecular structure (monomeric and dimeric structure) investigation of 2-[(2-hydroxyphenyl) carbonyloxy]benzoic acid by DFT method: A combined experimental and theoretical study, J. Mol. Struct, 1038, 145-162 (2013). DOI: $10.1016 /$ j.molstruc.2013.01.043

[80] N. G. Haress, F. Al-Omary, A. A. El-Emam, Y. S. Mary, C. Y. Panicker, A. A. Al-Saadi, J. A. War, C. Van Alse- noy, Spectroscopic investigation (FT-IR and FTRaman), vibrational assignments, HOMO-LUMO analysis and molecular docking study of 2-(adamantan-1-yl)5-(4-nitrophenyl)-1,3,4-oxadiazole, Spectrochim. Acta A 135, 973-983 (2014). DOI: 10.1016/j.saa.2014.07.077

[81] T. Karthick, V. Balachandran, S. Perumal, Spectroscopic investigations, molecular interactions, and molecular docking studies on the potential inhibitor "thiophene-2carboxylicacid", Spectrochim. Acta A 141, 104-112 (2015). DOI: $10.1016 /$ j.saa.2015.01.025

[82] R. Huey, G. M. Morris, A. J. Olson, D. S. Goodsell, A semiempirical free energy force field with charge-based desolvation, J. Comput. Chem. 28, 1145-1152 (2007). DOI: $10.1002 /$ jcc. 20634

[83] W. L. Delano, The PyMOL Molecular Graphics System, Version 1.8 Schrödinger, LLC (2012). 